\title{
Synthesis and antimycobacterial activity of disubstituted benzyltriazoles
}

\author{
Frans J. Smit ${ }^{1} \cdot$ Ronnett Seldon ${ }^{2} \cdot$ Janine Aucamp ${ }^{3} \cdot$ Audrey Jordaan $^{4} \cdot$ Digby F. Warner $\mathbb{C}^{4,5,6} \cdot$ David D. N'Da $\mathbb{1}^{3}$
}

Received: 22 July 2019 / Accepted: 5 October 2019 / Published online: 23 October 2019

(c) Springer Science+Business Media, LLC, part of Springer Nature 2019

\begin{abstract}
The increasing prevalence of multidrug-resistant strains of Mycobacterium tuberculosis (Mtb), the pathogen of human tuberculosis (TB), serves as a strong incentive for the discovery and development of new agents for the treatment of this plight. In search for such drugs, we investigated a series of benzyltriazole derivatives. We herein report the design, synthesis and biological activity of disubstituted benzyltriazoles against the human virulent H37Rv strain of $M t b$ as well as the toxicity on human embryonic kidney (HEK-293) cells. The derivative $\mathbf{2 1}$ featuring trifluoromethyl substituent in para position on the phenyl ring and $n$-butyl chain in position 4 on the triazole ring was the most active with $\mathrm{MIC}_{90}$ and $\mathrm{MIC}_{99}$ values of 1.73 and $3.2 \mu \mathrm{M}$, respectively, in the albumin-free medium. It also displays high selectivity towards bacteria growth inhibition (SI > 58), thus stands as a better hit for further investigation, including lead optimization, DMPK parameters determination and assessment of its activity in animal models.
\end{abstract}

\section{Graphical Abstract}

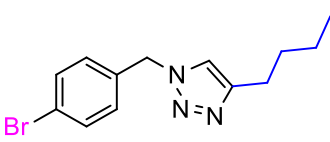

11

GAST/Fe MIC Mo $_{90}(\mu \mathrm{M}) 2.78$

GAST/Fe MIC Mg $_{99}(\mu \mathrm{M}) 3.82$

7H9/ADC MIC 90

HEK-293

cLoP

$\mathrm{SI}$<smiles>CCCCc1cn(Cc2ccc(C(F)(F)F)cc2)nn1</smiles>

21

1.73

3.19

$>125$

$>100$

4.3

$>58$

Disclaimer: Any opinions, findings and conclusions, or recommendations expressed in this material are those of the authors and therefore the NRF does not accept any liability in regard thereto.

Supplementary information The online version of this article (https:// doi.org/10.1007/s00044-019-02458-7) contains supplementary material, which is available to authorized users.

David D. N'Da

david.nda@nwu.ac.za

1 Pharmaceutical Chemistry, School of Pharmacy, North-West University, Potchefstroom 2520, South Africa

2 H3D Drug Discovery and Development Centre, Potchefstroom 2520, South Africa

3 Centre of Excellence for Pharmaceutical Sciences, North-West University, Potchefstroom 2520, South Africa
4 SAMRC/NHLS/UCT Molecular Mycobacteriology Research Unit, Division of Medical Microbiology, Department of Pathology, University of Cape Town, Cape Town, South Africa

5 Institute of Infectious Disease and Molecular Medicine, University of Cape Town, Rondebosch 7701, South Africa

6 Wellcome Centre for Infectious Diseases Research in Africa, University of Cape Town, Rondebosch 7701, South Africa 
Keywords Tuberculosis $\cdot$ TB $\cdot$ Drug discovery $\cdot$ Benzyltriazole $\cdot$ Click chemistry

\section{Introduction}

Tuberculosis (TB) is a major health challenging problem worldwide. According to the latest statistics of the World Health Organization (WHO), TB, alongside human immunodeficiency virus (HIV), malaria, dengue and hepatitis (WHO 2018a), are the leading infectious diseases causing death worldwide. In 2017 alone 10 million people contracted TB and 1.6 million fatalities were registered, of which more than $95 \%$ occurred in low- and middle-income countries (WHO 2018b). The majority of the estimated incidence cases occurred in South-East Asia (44\%), and Africa (25\%) (WHO 2018b), making TB a disease of the developing world.

TB is a treatable disease. However, its treatment is long and complex and despite the availability of useful drugs and regimens, the disease still causes numerous fatalities annually. The existing antitubercular drugs, although of immense value in controlling the disease, have several limitations with the most important being the emergence of drug-resistant TB strains against the frontline drugs (Amir et al. 2014). Drug-resistant TB occurs when Mycobacterium tuberculosis $(M t b)$, the causative agent of human TB, becomes resistant to at least one of the first-line anti-TB drugs. Multidrug-resistant TB (MDR-TB) occurs when $M t b$ becomes resistant to the two most effective anti-TB drugs, isoniazid (INH) and rifampicin (RIF) (CDC 2018a). In addition, MDR-TB patients with further resistance to any fluoroquinolone and at least one of the three injectable second-line drugs (capreomycin, kanamycin or amikacin) is classified as extensively drug-resistant TB (XDR-TB) (CDC 2018a). In 2017 alone, the WHO revealed an estimate of 558,000 new cases of RIF resistance with $82 \%$ suffering from MDR-TB, of which $8.5 \%$ were reported as XDR-TB cases. To make matters worse, only 55\% of MDR-TB cases were treated successfully during that year (WHO 2018b). The advent of drug-resistant TB has made the treatment and cure of TB even more complicated.

Drug susceptible TB chemotherapy is a lengthy regimen, spanning over a 6-9-month period and consisting of a multidrug therapy that combines four anti-TB drugs, namely RIF, INH, pyrazinamide (PYZ) and ethambutol (CDC 2018b). In contrast, the treatment of drug-resistant TB requires a regimen that consists of at least five effective TB agents during the intensive phase. The combination should comprise PYZ and four core second-line TB agents, namely one fluoroquinolone, one aminoglycoside, one thionamide (ethionamide or prothionamide), and either cycloserine or terizidone (WHO 2016).
However, apart from drug resistance the current anti-TB drugs possess several limitations, including a high prevalence of adverse side effects and the inability to act upon latent forms of the Mtb bacillus (Amir et al. 2014), leading to patient non-adherence and ultimately to a discontinuation of therapy with further implications. Adverse side effects caused by drug therapy may incur substantial additional costs due to outpatient visits, additional tests and, in more serious cases, hospitalisation. Second-line agents often have more severe effects, are less effective, have worse toxicity profiles and are more expensive (Kumar et al. 2014a; Yee et al. 2003). The treatment duration of second-line agents is often prolonged by 2 years (Kumar et al. 2014a), adding additional challenges to patient compliance and resulting in a high risk of treatment failure and relapse (Yee et al. 2003).

An additional factor driving up the TB death toll is coinfection with HIV. In 2017 TB caused 300,000 deaths among HIV-positive people (WHO 2018b), while HIV coinfection accounted for 900,000 new cases to the global TB morbidity (WHO 2018b). The resistance against mainstay anti-TB drugs, such as isoniazid, RIF and PYZ that have been attributed to parasite genetic mutations (Jureen et al. 2008; Seifert et al. 2015; Zaw et al. 2018), have rendered the disease intractable. Thus, the emergence of MDR strains of $M t b$ threatens the global strategy to eradicate the disease by 2030 (WHO 2018b). Moreover, roughly one-third of the world's population carries a latent form of TB-meaning these individuals have been infected but are not ill and cannot transmit the disease yet. However, the danger of passing the infection on to another person can occur anytime.

Altogether, these facts underscore the need for new antiTB drugs to treat all the forms of the diseases. In recent years efforts have focused on the discovery of new anti-TB agents with divergent, unique structures and mechanisms of action different from those of currently used drugs, in order to treat TB to achieve effective disease control (Sajja et al. 2017).

A well-known privileged nucleus that has drawn much attention in drug discovery is the triazole core (Dheer et al. 2017). The 1,2,3-triazole is a five-member $N$-heterocyclic compound (Ali et al. 2017) that has attracted significant attention due to the wide range of biological properties of compounds containing this moiety, including antitubercular (Boechat et al. 2011b), antifungal (Dai et al. 2015), anti-HIV (Mohammed et al. 2016), antimalarial (Kumar et al. 2014b; Singh et al. 2017) and antiinflammatory activity (Shafi et al. 2012). This triazole scaffold possesses hydrogen bonding capability, moderate 
Fig. 1 Structures of 1,2,3triazole and benzyltriazole derivatives<smiles>NC(C(=O)N[C@H]1C(=O)N2C(C(=O)O)C(CSC3=CNNN3)CSC12)c1ccc(O)cc1</smiles>

1,2,3-Triazole<smiles>NC(=O)c1cn(Cc2c(F)cccc2F)nn1</smiles>

Rufinamide<smiles>CCCCCCCCc1cn(CC(=O)OCC)nn1</smiles>

dipole character, rigidity and stability under in vivo conditions that all together are responsible for the enhanced biological properties of compounds containing that scaffold (Zhang et al. 2017). Interestingly, some triazolecontaining compounds and the mainstay anti-TB drug, $\mathrm{INH}$, share a similar mechanism of action as they inhibit microbial cell wall synthesis by blocking lipid biosynthesis (Kumar et al. 2014a; Zhang et al. 2017), presumably by targeting the enoyl-acyl carrier protein (PDB ID: 1ZID) that is essential for the fatty acid (mycolic acids) synthase system (FAS-II) in mycobacterial cells (Rozwarski et al. 1998). Drugs currently in the market that possess the 1,2,3-triazole moiety include cefatrizine and tazobactam (antibiotics) (Ali et al. 2017; Zhang et al. 2017), TSAO (anti-HIV) (Zhang et al. 2017), and carboxyamidotriazole (CAI) (anticancer) (Fig. 1) (Ali et al. 2017; Zhang et al. 2017). Benzofuran salicylic acid derivative (I-A09), a synthesized 1,2,3-triazole derivative, is currently the lead anti-TB agent under clinical evaluation and may be used to treat TB in the near future (Ali et al. 2017; Zhang et al. 2017). The triazole derivatives A (Labadie et al. 2011) and B (Boechat et al. 2011a) (Fig. 1) have shown activity against different pathogenic and opportunistic mycobacteria, including M. avium and Mtb.
The benzyl moiety also plays an important role in biochemical and pharmacological processes and is herein envisaged as a partner to 1,2,3-triazole. Incorporated into a quinoxaline scaffold it acts as a DNA intercalation switch, resulting in anticancer properties (Mahata et al. 2016). On the other hand, when incorporated into polyamine it contributes to the inhibition of Plasmodium falciparum's essential polyamine biosynthetic pathway (El Bissati et al. 2019). Benzyl containing compounds display a wide array of pharmacological activities, including antitubercular (Cheng et al. 2019; Gallardo-Macias et al. 2019; Zhang et al. 2018), antimicrobial (Belz et al. 2013; Swetha et al. 2019), antimalarial (Courtens et al. 2018; El Bissati et al. 2019; Tahghighi et al. 2018) and antifungal (Ballari et al. 2017; Belz et al. 2013). Clinical pharmacological drugs wherein the 1,2,3-triazole and benzyl scaffolds are conjugated to form the benzyltriazole (BZT) moiety include the anticancer agent CAI and the antiepileptic rufinamide (Fig. 1).

The mycobacterium cell wall is lipophilic in nature (Suresh et al. 2014); therefore, lipophilicity is an imperative consideration in the design of active antitubercular molecules. Thus, in the midst of addressing the drug-resistance issue through increased activity, the 1,2,3-triazole scaffold was directly anchored to the benzyl moiety in position $\mathrm{N}-1$ 
to generate novel BZT derivatives with varying lipophilicity imparted by the substituents on the rings. We herein report the synthesis, in vitro antimycobacterial activities as well as cytotoxicity of these derivatives.

\section{Material and methods}

\section{Materials}

Solvents: acetonitrile (ACN), dichloromethane (DCM), hexane and methanol $(\mathrm{MeOH})$ were obtained from ACE Chemicals. Reagents, such as benzyl bromides, alkynes, copper sulfate pentahydrate $\left(\mathrm{CuSO}_{4} \cdot 5 \mathrm{H}_{2} \mathrm{O}\right)$, sodium azide $\left(\mathrm{NaN}_{3}\right)$, sodium ascorbate (NaAsc), $\beta$-cyclodextrin ( $\beta$-CD) and 3-(phenylmercapto)propylene-1, were all acquired from Sigma-Aldrich South Africa.

\section{General procedures}

The ${ }^{1} \mathrm{H}$ and ${ }^{13} \mathrm{C}$ nuclear magnetic resonance (NMR) spectra were recorded on a Bruker Advance ${ }^{\mathrm{TM}}$ III 600 spectrometer at a frequency of 600 and $150.913 \mathrm{MHz}$, respectively, in $\mathrm{CDCl}_{3}-d$. Chemical shifts are reported in parts per million $\delta$ (ppm), with the residual protons of the solvent as reference. The splitting pattern abbreviations are as follows: singlet (s), doublet (d), doublet of doublet (dd), doublet of triplets $(\mathrm{dt})$, triplet ( $\mathrm{t})$, triplet of doublets (td), triplet of triplets ( $\mathrm{tt})$, quartet of doublets (qd) and multiplet (m).

High resolution mass spectrometry (HRMS) was recorded on a Bruker MicroTOF Q II mass spectrometer, equipped with an APCI source, set at 200 or $180^{\circ} \mathrm{C}$, respectively, using Bruker Compass Data Analysis 4.0 software. A full scan from $50-1500 \mathrm{~m} / \mathrm{z}$ was performed at a capillary voltage of $4500 \mathrm{~V}$, an end plate offset voltage of $-500 \mathrm{~V}$, with the nebulizer set at 1.6 and $0.4 \mathrm{Bar}$, respectively, and a collision cell RF voltage of $100 \mathrm{Vpp}$.

Infrared (FTIR) spectra were recorded on a Bruker Alpha-P FTFTIR instrument. Thin layer chromatography (TLC) was performed using silica gel plates $\left(60 \mathrm{~F}_{254}\right)$ obtained from Merck (Johannesburg, South Africa). TLC was utilized to monitor the progress of reactions and visualization of spots was done under UV-light $(254 \mathrm{~nm})$ as well as iodine. Column chromatography was performed using silica gel (230-400 mesh, Sigma-Aldrich).

High performance liquid chromatography (HPLC) analysis of all final compounds were performed to determine purity. An Agilent 1100 HPLC system, equipped with a quaternary pump and an Agilent 1100 series diode array detector, was utilized. HPLC grade acetonitrile (Merck) and Milli-Q water (Millipore) were used for chromatography. A Venusil XBP C18 column $(4.60 \times 150 \mathrm{~mm}, 5 \mu \mathrm{m})$ with an initial mobile phase (70\% Milli-Q water: $30 \% \mathrm{ACN}$ ) was employed at a flow rate of $1 \mathrm{ml} / \mathrm{min}$. The concentration of $\mathrm{ACN}$ in the mobile phase was linearly increased over a period of $5 \mathrm{~min}$ to a final concentration of $85 \%$. The time allowed for equilibration between runs was $5 \mathrm{~min}$ and the duration of each HPLC run was $15 \mathrm{~min}$. The concentration of the test compounds injected varied $(20 \mu \mathrm{l}$ of $1 \mathrm{mM}-20 \mu \mathrm{l}$ of $0.25 \mathrm{mM}$ ). The eluent was monitored at wavelengths of 210, 254 and $300 \mathrm{~nm}$.

Furthermore, the following abbreviations are adopted, $\mathrm{C}_{\mathrm{Ar}}$ (carbon of aromatic ring), $\mathrm{H}_{\mathrm{Ar}}$ (hydrogen of aromatic ring), Tz (triazole) and BZT.

\section{Synthesis}

Benzyl halide (1 eq.) and the appropriate alkyne (1.2 eq.) were mixed in a round-bottomed flask. Tetrahydrofuran (THF; $5 \mathrm{ml}), \mathrm{MeOH}(5 \mathrm{ml})$ and distilled water $(5 \mathrm{ml})$ were added consecutively. To this stirring solution were added $\beta-\mathrm{CD}$ (60 mg, 0.02 eq.), NaAsc ( $240 \mathrm{mg}, 0.2$ eq.), $\mathrm{NaN}_{3}$ ( $230 \mathrm{mg}$, 1.2 eq.) and $\mathrm{CuSO}_{4} \cdot 5 \mathrm{H}_{2} \mathrm{O}(120 \mathrm{mg}, 0.2$ eq.) sequentially. The solution was stirred until a white precipitate formed. If the solution turned black, an additional portion of sodium ascorbate $(50 \mathrm{mg}$ ) was added until the solution turned clear yellow. The reaction was followed by TLC. After completion, DCM $(50 \mathrm{ml})$ and saturated sodium bicarbonate $\left(\mathrm{NaHCO}_{3}\right.$; $50 \mathrm{ml}$ ) were added. The organic layer was separated and the aqueous phase was extracted with DCM $(3 \times 50 \mathrm{ml})$. The combined organic phase was washed with water $(3 \times 50 \mathrm{ml})$, dried over $\mathrm{MgSO}_{4}$, filtered off and rotated to dryness in vacuo, resulting in a hot oil residue. Hexane $(50 \mathrm{ml})$ was added to the hot oil and the supernatant was decanted into a flat bottom flask. This was repeated two times, the combined extracts cooled at room temperature and then allowed to crystallize at 0 to $-4{ }^{\circ} \mathrm{C}$ to afford the target compound.

\section{1-benzyl-4-butyl-1H-1,2,3-triazole 1}

Light off-white crystals; yield (68\%); m.p. $61.5-64.1{ }^{\circ} \mathrm{C}$; FTIR $v_{\max }: 3113(=\mathrm{C}-\mathrm{H}, \mathrm{Tz}), 3062\left(=\mathrm{C}-\mathrm{H}_{\mathrm{Ar}}\right), 1568(\mathrm{C}=\mathrm{C}$, $\mathrm{Tz}), 1492(\mathrm{~N}=\mathrm{N}) \mathrm{cm}^{-1} ;{ }^{1} \mathrm{H}$ NMR $\left(600 \mathrm{MHz}, \mathrm{CDCl}_{3}\right) \delta$ 7.37-7.28 (m, 3H, $2 \mathrm{H}-9$ and H-5), 7.26-7.12 (m, 3H, $2 \mathrm{H}-8$ and $\mathrm{H}-10), 5.46(\mathrm{~s}, 2 \mathrm{H}, \mathrm{H}-6), 2.66(\mathrm{t}, J=7.6 \mathrm{~Hz}, 2 \mathrm{H}, \mathrm{H}-11)$, 1.65-1.53 (m, 2H, H-12), 1.38-1.28 (m, 2H, H-13), 0.88 (t, $J=7.4 \mathrm{~Hz}, 3 \mathrm{H}, \mathrm{H}-14) ;{ }^{13} \mathrm{C}$ NMR $\left(151 \mathrm{MHz}, \mathrm{CDCl}_{3}\right) \delta$ 134.92 (C-7), 129.00 (C-9), 128.56 (C-8), 127.92 (C-10), 53.99 (C-6), 31.42 (C-12), 25.34 (C-11), 22.27 (C-13), 13.76 (C-14); HRMS (APCI) $m / z:[\mathrm{M}+\mathrm{H}]^{+} 216.1507$ (Calc. for $\mathrm{C}_{13} \mathrm{H}_{18} \mathrm{~N}_{3}$ : 216.1501); purity (HPLC): $96 \%$.

\section{1-benzyl-6-hexyl-1H-1,2,3-triazole 2}

Fluffy off-white crystals; yield (87\%); m.p $60.3-61.1^{\circ} \mathrm{C}$. FTIR $v_{\max }: 3036(=\mathrm{C}-\mathrm{H}, \mathrm{Tz}), 2917(-\mathrm{C}-\mathrm{H}), 1493(\mathrm{~N}=\mathrm{N})$ 
$\mathrm{cm}^{-1} ;{ }^{1} \mathrm{H}$ NMR $\left(600 \mathrm{MHz}, \mathrm{CDCl}_{3}\right) \delta$ 7.37-7.27 (m, 3H, 2 $\mathrm{H}-9$ and H-5), 7.27-7.15 (m, 3H, $2 \mathrm{H}-8$ and H-10), 5.47 (s, $2 \mathrm{H}, \mathrm{C}-6), 1.37-1.19$ (m, 6H, H-13, -14 and 15$), 0.86-0.78$ (m, 3H, H-16); ${ }^{13} \mathrm{C}$ NMR (151 MHz, $\left.\mathrm{CDCl}_{3}\right) \delta 134.94(\mathrm{C}-$ 7), 128.97 (C-9), 128.54 (C-8), 127.90 (C-10), 54.08 (C-6), 31.48 (C-12), 25.69 (C-11), 13.99 (C-16); HRMS (APCI) $m / z:[\mathrm{M}+\mathrm{H}]^{+} 244.1825$ (calc. for $\mathrm{C}_{15} \mathrm{H}_{22} \mathrm{~N}_{3}$ : 244.1814); purity (HPLC): $95 \%$.

\section{1-benzyl-4-octyl-1H-1,2,3-triazole 3}

Light yellow crystals; yield (74\%); m.p. 71.6-72.2 ${ }^{\circ} \mathrm{C}$; FTIR $v_{\max }: 3063(=\mathrm{C}-\mathrm{H}, \mathrm{Tz}), 2916(-\mathrm{C}-\mathrm{H}), 1493(\mathrm{~N}=\mathrm{N}), 1456$ $(\mathrm{N}=\mathrm{N}) \mathrm{cm}^{-1} ;{ }^{1} \mathrm{H}$ NMR $\left(600 \mathrm{MHz}, \mathrm{CDCl}_{3}\right) \delta 7.39-7.25(\mathrm{~m}$, $3 \mathrm{H}, 2 \mathrm{H}-9$ and H-5), 7.25-7.11 (m, 3H, $2 \mathrm{H}-8$ and $\mathrm{H}-10)$, 5.47 (s, 2H, H-6), 1.35-1.15 (m, 10H, H-13, -14, -15, -16, -17), 0.84 (t, $J=7.0 \mathrm{~Hz}, 3 \mathrm{H}, \mathrm{H}-18) ;{ }^{13} \mathrm{C} \mathrm{NMR}(151 \mathrm{MHz}$, $\left.\mathrm{CDCl}_{3}\right) \delta 134.93$ (C-7), 128.95 (C-9), 128.53 (C-8), 127.90 (C-10), 54.91-52.73 (C-6), 31.75 (C-12), 25.69 (C-11), 22.58 (C-17), 14.03 (C-18); HRMS (APCI) $m / z:[\mathrm{M}+\mathrm{H}]^{+} 272.2127$ (calc. for $\mathrm{C}_{17} \mathrm{H}_{26} \mathrm{~N}_{3}$ : 272.2127); purity (HPLC): $96 \%$.

\section{1-benzyl-4-decyl-1H-1,2,3-triazole 4}

Light yellow crystals; yield (64\%); m.p. $80.5-81.2^{\circ} \mathrm{C}$; FTIR $v_{\max }$ : 3063 (=C-H, Tz), $2915(-\mathrm{C}-\mathrm{H}), 1468(\mathrm{~N}=\mathrm{N})$ $\mathrm{cm}^{-1} ;{ }^{1} \mathrm{H}$ NMR $\left(600 \mathrm{MHz}, \mathrm{CDCl}_{3}\right) \delta$ 7.37-7.27 (m, 3H, 2 H-9 and H-5), 7.26-7.08 (m, 3H, 2 H-8 and H-10), 5.47 (s, 2H, H-6), 1.35-1.14 (m, 14H, H-13, -14, -15, -16, -17, -18 and -19$), 0.85(\mathrm{t}, J=7.0 \mathrm{~Hz}, 3 \mathrm{H}, \mathrm{H}-20) ;{ }^{13} \mathrm{C} \quad \mathrm{NMR}$ $\left(151 \mathrm{MHz}, \mathrm{CDCl}_{3}\right) \delta 134.92$ (C-7), 129.00 (C-9), 128.58 (C-8), 127.94 (C-10), 54.57-52.51 (C-6), 31.85 (C-12), 25.68 (C-11), 22.64 (C-19), 14.08 (C-20); HRMS (APCI) $m / z:[\mathrm{M}+\mathrm{H}]^{+} 300.2449$ (calc. for $\mathrm{C}_{19} \mathrm{H}_{30} \mathrm{~N}_{3}$ : 300.2440); purity (HPLC): $97 \%$.

\section{1-benzyl-4-[(phenylsulfanyl)methyl]-1H-1,2,3-triazole 5}

White crystals, yield (66\%); m.p. $78.2-81.7^{\circ} \mathrm{C}$; FTIR $v_{\max }$ : $3139(=\mathrm{C}-\mathrm{H}, \mathrm{Tz}), 1582(\mathrm{C}=\mathrm{C}, \mathrm{Tz}), 1454(\mathrm{~N}=\mathrm{N}) \mathrm{cm}^{-1} ;{ }^{1} \mathrm{H}$ NMR $\left(600 \mathrm{MHz}, \mathrm{CDCl}_{3}\right) \delta$ 7.35-7.23 (m, 6H, $2 \mathrm{H}-9,2 \mathrm{H}-$ 14, H-8 and H-5), 7.20 (t, $J=7.4 \mathrm{~Hz}, 2 \mathrm{H}, \mathrm{H}-13$ ), 7.17-7.11 (m, 3H, H-10, H-15), 5.42 (s, 2H, H-6), 4.19 (s, 2H, H-11); ${ }^{13} \mathrm{C} \mathrm{NMR}\left(151 \mathrm{MHz}, \mathrm{CDCl}_{3}\right.$ ) $\delta 135.16$ (C-7), 134.51 (C-13), 129.80 (C-14, -10), 128.85 (C-8), 127.86 (C-15), 126.49 (C6), 28.93 (C-11); HRMS (APCI) $m / z:[\mathrm{M}+\mathrm{H}]^{+} 282.1057$ (calc. for $\mathrm{C}_{16} \mathrm{H}_{16} \mathrm{~N}_{3} \mathrm{~S}$ : 282.1065); purity (HPLC): $91 \%$.

\section{4-butyl-1-[(4-methylphenyl)methyl]-1H-1,2,3-triazole 6}

Light yellow powder; yield (75\%); m.p.: $64.2-65.3{ }^{\circ} \mathrm{C}$; FTIR $v_{\max }: 3115(=\mathrm{C}-\mathrm{H}, \mathrm{Tz}), 3063\left(=\mathrm{C}-\mathrm{H}_{\mathrm{Ar}}\right), 1616(\mathrm{C}=\mathrm{C}$, $\mathrm{Tz}), 1456(\mathrm{~N}=\mathrm{N}) \mathrm{cm}^{-1} ;{ }^{1} \mathrm{H} \mathrm{NMR}\left(600 \mathrm{MHz}, \mathrm{CDCl}_{3}\right) \delta$
7.13 (q, $J=8.2 \mathrm{~Hz}, 5 \mathrm{H}, 2 \mathrm{H}-9,2 \mathrm{H}-8, \mathrm{H}-5), 5.41$ (s, 2H, H6), 2.32 (s, 3H, H-10), 1.38-1.26 (m, 2H, H-17), 0.92-0.82 (m, 3H, H-18); ${ }^{13} \mathrm{C}$ NMR $\left(151 \mathrm{MHz}, \mathrm{CDCl}_{3}\right) \delta 138.46(\mathrm{C}-$ 10), 131.88 (C-7), 129.63 (C-9), 127.98 (C-5), 54.57-51.95 (C-6), 25.35 (C-11), 22.26 (C-17), 21.09 (C-10'), 13.75 (C-18); HRMS (APCI) $m / z:[\mathrm{M}+\mathrm{H}]^{+} 230.1650$ (calc. for $\mathrm{C}_{14} \mathrm{H}_{20} \mathrm{~N}_{3}$ : 230.1657); purity (HPLC): $95 \%$.

\section{4-hexyl-1-[(4-methylphenyl)methyl]-1H-1,2,3-triazole 7}

Cream coloured powder; yield (63\%); m.p.: 65.5-72.0 ${ }^{\circ} \mathrm{C}$; FTIR $v_{\max }: 3114(=\mathrm{C}-\mathrm{H}, \mathrm{Tz}), 3063(=\mathrm{C}-\mathrm{H}), 1615(\mathrm{C}=\mathrm{C}$, $\mathrm{Tz}), 1455(\mathrm{~N}=\mathrm{N}) \mathrm{cm}^{-1} ;{ }^{1} \mathrm{H}$ NMR $\left(600 \mathrm{MHz}, \mathrm{CDCl}_{3}\right) \delta$ 7.18-7.08 (m, 5H, 5H, 2 H-9, 2 H-8, H-5), 5.42 (s, 2H, H6), 2.33 (s, 3H, H-10'), 1.36-1.19 (m, 6H, H-13, -14, -25), $0.84(\mathrm{t}, J=6.6 \mathrm{~Hz}, 3 \mathrm{H}, \mathrm{H}-6) ;{ }^{13} \mathrm{C} \mathrm{NMR}\left(151 \mathrm{MHz}, \mathrm{CDCl}_{3}\right)$ ठ 138.50 (C-10), 131.89 (C-7), 129.64 (C-8), 128.02 (C-9, -5), 31.50 (C-12), 25.71 (C-11), 22.49 (C-15), 21.10 (C$\left.10^{\prime}\right), 14.00$ (C-16); HRMS (APCI) $m / z:[\mathrm{M}+\mathrm{H}]^{+} 258.1957$ (calc. for $\mathrm{C}_{16} \mathrm{H}_{24} \mathrm{~N}_{3}$ : 258.1970); purity (HPLC): $93 \%$.

\section{4-octyl-1-[(4-methylphenyl)methyl]-1H-1,2,3-triazole 8}

White powder; yield (68\%); m.p.: 69.5-70.4 ${ }^{\circ} \mathrm{C}$; FTIR $v_{\max }$ : 3113 (=C-H, Tz), $3064\left(=\mathrm{C}-\mathrm{H}_{\mathrm{Ar}}\right), 1615(\mathrm{C}=\mathrm{C}, \mathrm{Tz}), 1468$ $(\mathrm{N}=\mathrm{N}) \mathrm{cm}^{-1} ;{ }^{1} \mathrm{H}$ NMR $\left(600 \mathrm{MHz}, \mathrm{CDCl}_{3}\right) \delta 7.13(\mathrm{dd}, J=$ 17.9, 7.9 Hz, 5H, 2 H-9, 2 H-8, H-5), 5.42 (s, 2H, H-6), 2.33 (s, 3H, H-10'), 1.38-1.11 (m, 10H, H-13, -14, -15, -16, -17), 0.84 (t, $J=7.0 \mathrm{~Hz}, 3 \mathrm{H}, \mathrm{H}-18) ;{ }^{13} \mathrm{C} \mathrm{NMR}(151 \mathrm{MHz}$, $\left.\mathrm{CDCl}_{3}\right) \delta 138.50(\mathrm{C}-10), 131.88(\mathrm{C}-7), 129.63$ (C-8), 128.03 (C-9), 31.78 (C-12), 25.71 (C-11), $21.09\left(\mathrm{C}-10^{\prime}\right)$, $14.06 \mathrm{C}-18$ ); HRMS (APCI) $m / z:[\mathrm{M}+\mathrm{H}]^{+} 286.2260$ (calc. for $\mathrm{C}_{18} \mathrm{H}_{28} \mathrm{~N}_{3}$ : 286.2283); purity (HPLC): $96 \%$.

\section{4-decyl-1-[(4-methylphenyl)methyl]-1H-1,2,3-triazole 9}

Light yellow solid; yield (64\%); m.p.: 76.1-77.6 ${ }^{\circ}$; FTIR $v_{\max }: 2915(=\mathrm{C}-\mathrm{H}, \mathrm{Tz}), 2848,1514(\mathrm{C}=\mathrm{C}, \mathrm{Tz}), 1468$ $(\mathrm{N}=\mathrm{N}) \mathrm{cm}^{-1} ;{ }^{1} \mathrm{H}$ NMR $\left(600 \mathrm{MHz}, \mathrm{CDCl}_{3}\right) \delta 7.18(\mathrm{q}, J=$ 8.2 Hz, 5H, 2 H-9, 2 H-8, H-5), 5.46 (s, 2H, H-6), 2.69 (t, $J=7.7 \mathrm{~Hz}, 2 \mathrm{H}, \mathrm{H}-11), 2.37$ (s, 3H, H-10'), 1.64 (dd, $J=$ 14.5, 7.3 Hz, 2H, H-12), 0.89 (t, $J=7.1 \mathrm{~Hz}, 3 \mathrm{H}, \mathrm{H}-20) ;{ }^{13} \mathrm{C}$ NMR (151 MHz, $\left.\mathrm{CDCl}_{3}\right) \delta 138.53$ (C-10), 131.97 (C-7), 129.72 (C-8), 128.05 (C-9), 120.40 (C-5), 53.85 (C-6), 25.75 (C-11), $22.71\left(-\mathrm{CH}_{2}-\mathrm{CH}_{3}\right), 21.18\left(\mathrm{C}-10^{\prime}\right), 14.15$ (C-20); HRMS (APCI) $m / z:[\mathrm{M}+\mathrm{H}]^{+} 314.2575$ (calc. for $\mathrm{C}_{20} \mathrm{H}_{32} \mathrm{~N}_{3}$ : 314.2596 ); purity (HPLC): $80 \%$.

1-[(4-methylphenyl)methyl]-4-[(phenylsulfanyl)methyl]-1H1,2,3-triazole 10

Gold powder; yield (74\%); m.p.: $80.9-82.4{ }^{\circ} \mathrm{C}$; ${ }^{1} \mathrm{H}$ NMR (600 MHz, $\mathrm{CDCl}_{3}$ ) $\delta$ 7.30-7.10 (m, H-5, -8, -9, -13, -14, -15), 
5.43 (s, 2H, H-6), 4.22 (s, 2H, H-11), 2.37 (s, 3H, H-10'); ${ }^{13} \mathrm{C}$ NMR $\left(151 \mathrm{MHz}, \mathrm{CDCl}_{3}\right) \delta 138.66(\mathrm{C}-10), 135.37$ (C-7), 126.52 (C-5), 54.05 (C-6), 29.01 (C-11), 21.19 $\left(\mathrm{C}-10^{\prime}\right)$; HRMS (APCI) $\mathrm{m} / \mathrm{z}:[\mathrm{M}+\mathrm{H}]^{+} 296.1194$ (calc. for $\mathrm{C}_{17} \mathrm{H}_{18} \mathrm{~N}_{3} \mathrm{~S}$ : 296.1221); purity (HPLC): $92 \%$.

\section{1-[(4-bromophenyl)methyl]-4-butyl-1H-1,2,3-triazole 11}

White powder; yield (95\%); m.p. 76.4-77. ${ }^{\circ} \mathrm{C}$; FTIR $v_{\max }$ : $2918 \quad(=\mathrm{C}-\mathrm{H}, \quad \mathrm{Tz}), \quad 2853, \quad 1488 \quad(\mathrm{C}=\mathrm{C}, \quad \mathrm{Tz}), \quad 1452$ $(\mathrm{N}=\mathrm{N}) \mathrm{cm}^{-1} ;{ }^{1} \mathrm{H} \mathrm{NMR}\left(600 \mathrm{MHz}, \mathrm{CDCl}_{3}\right) \delta 7.51-7.41(\mathrm{~m}$, $2 \mathrm{H}, \mathrm{H}-9), 7.14(\mathrm{~d}, J=27.6 \mathrm{~Hz}, 1 \mathrm{H}, \mathrm{H}-5), 7.10(\mathrm{~d}, J=$ $8.4 \mathrm{~Hz}, 2 \mathrm{H}, \mathrm{H}-8), 5.42$ (s, 2H, H-6), 1.36-1.27 (m, 2H, H12), 0.89 (t, $J=7.4 \mathrm{~Hz}, 3 \mathrm{H}, \mathrm{H}-14) ;{ }^{13} \mathrm{C}$ NMR $(151 \mathrm{MHz}$, $\mathrm{CDCl}_{3}$ ) $\delta 149.07$ (C-4), 133.96 (C-7), 132.18 (C-9), 129.53 (C-8), 122.71 (C-10), 120.43 (C-5), 53.26 (C-6), 25.31 (C-11), 22.27 (C-13), 13.76 (C-14); HRMS (APCI) m/z: $[\mathrm{M}+\mathrm{H}]^{+} 294.0619$ (calc. for $\mathrm{C}_{13} \mathrm{H}_{17} \mathrm{BrN}_{3}$ : 294.0606); purity (HPLC): $93 \%$.

\section{1-[(4-bromophenyl)methyl]-4-hexyl-1H-1,2,3-triazole 12}

Beige powder; yield (69\%); m.p. $76.3-76.9^{\circ} \mathrm{C}$; FTIR $v_{\max }$ : $2922(=\mathrm{C}-\mathrm{H}, \mathrm{Tz}), 2853,1488(\mathrm{C}=\mathrm{C}, \mathrm{Tz}), 1465(\mathrm{~N}=\mathrm{N}) \mathrm{cm}^{-1}$; ${ }^{1} \mathrm{H}$ NMR $\left(600 \mathrm{MHz}, \mathrm{CDCl}_{3}\right) \delta$ 7.51-7.42 (m, 2H, H-9), 7.16 (s, 1H, H-5), 7.10 (t, $J=5.4 \mathrm{~Hz}, 2 \mathrm{H}, \mathrm{H}-8), 5.42$ (s, 2H, $\mathrm{H}-6), 1.61$ (dt, $J=15.4,7.6 \mathrm{~Hz}, 2 \mathrm{H}, \mathrm{H}-12), 1.36-1.17$ (m, 6H, H-13, -14, -15); ${ }^{13} \mathrm{C}$ NMR (151 MHz, $\left.\mathrm{CDCl}_{3}\right) \delta$ 149.12 (C-4), 133.97 (C-7), 132.20 (C-8), 129.52 (C-9), 122.73 (C-10), 120.44 (C-5), 53.27 (C-6), 14.01 (C-16); HRMS (APCI) m/z: $[\mathrm{M}+\mathrm{H}]^{+} 322.0936$ (calc. for $\mathrm{C}_{15} \mathrm{H}_{21} \mathrm{BrN}_{3}$ : 322.0919 ); purity (HPLC): $95 \%$.

\section{1-[(4-bromophenyl)methyl]-4-octyl-1H-1,2,3-triazole 13}

Tan powder; yield (59\%); m.p. $72.3-73.3^{\circ} \mathrm{C}$; FTIR $v_{\max }$ : $2916 \quad(=\mathrm{C}-\mathrm{H}, \quad \mathrm{Tz}), \quad 2849, \quad 1488 \quad(\mathrm{C}=\mathrm{C}, \quad \mathrm{Tz}), 1466$ $(\mathrm{N}=\mathrm{N}) \mathrm{cm}^{-1} ;{ }^{1} \mathrm{H}$ NMR $\left(600 \mathrm{MHz}, \mathrm{CDCl}_{3}\right) \delta 7.50-7.41(\mathrm{~m}$, 2H, H-9), 7.17 (d, $J=10.2 \mathrm{~Hz}, 1 \mathrm{H}, \mathrm{H}-5), 7.10$ (d, $J=$ $8.4 \mathrm{~Hz}, 2 \mathrm{H}, \mathrm{H}-8), 5.42$ (s, 2H, H-6), 1.65-1.54 (m, 2H, H12), $0.86(\mathrm{dt}, J=14.1,7.4 \mathrm{~Hz}, 3 \mathrm{H}, \mathrm{H}-18) ;{ }^{13} \mathrm{C}$ NMR $\left(151 \mathrm{MHz}, \mathrm{CDCl}_{3}\right) \delta 149.78$ (C-4), 133.99 (C-7), 132.18 (C-8), 129.52 (C-9), 122.71 (C-10), 120.43 (C-5), 53.25 (C6), 14.07 (C-18); HRMS (APCI) $\mathrm{m} / z:[\mathrm{M}+\mathrm{H}]^{+} 350.1233$ (calc. for $\mathrm{C}_{17} \mathrm{H}_{25} \mathrm{BrN}_{3}$ : 350.1232 ); purity (HPLC): $96 \%$.

\section{1-[(4-bromophenyl)methyl]-4-decyl-1H-1,2,3-triazole 14}

Beige powder; yield (81\%); m.p. 84.3-85. ${ }^{\circ} \mathrm{C}$; FTIR $v_{\max }$ : $3112(=\mathrm{C}-\mathrm{H}, \mathrm{Tz}), 3062\left(=\mathrm{C}-\mathrm{H}_{\mathrm{Ar}}\right), 1590(\mathrm{C}=\mathrm{C}, \mathrm{Tz}), 1467$ $(\mathrm{N}=\mathrm{N}) \mathrm{cm}^{-1} ;{ }^{1} \mathrm{H}$ NMR $\left(600 \mathrm{MHz}, \mathrm{CDCl}_{3}\right) \delta 7.51-7.41(\mathrm{~m}$, 2H, H-9), 7.16 (s, 1H, H-5), 7.10 (d, $J=8.4 \mathrm{~Hz}, 2 \mathrm{H}, \mathrm{H}-8$ ), 5.42 (s, 2H, H-6), 1.64-1.54 (m, 2H, H-12), 1.35-1.15 (m,
14H, H-13, -14, -15, -16, -17, -18, -19), 0.85 (t, $J=7.1 \mathrm{~Hz}$, $\mathrm{H}-20) ;{ }^{13} \mathrm{C}$ NMR (151 MHz, $\left.\mathrm{CDCl}_{3}\right) \delta 149.14$ (C-4), 133.98 (C-7), 132.19 (C-8), 129.52 (C-9), 122.72 (C-10), 120.43 (C-5), 53.26 (C-6), $31.86\left(-\mathrm{CH}_{2}-\mathrm{CH}_{2}-\mathrm{CH}_{3}\right), 25.65$ (C-11), 14.08 (C-20); HRMS (APCI) $m / z:[\mathrm{M}+\mathrm{H}]^{+}$ 378.1536 (calc. for $\mathrm{C}_{19} \mathrm{H}_{29} \mathrm{BrN}_{3}$ : 378.1545); purity (HPLC): $97 \%$.

1-[(4-bromophenyl)methyl]-4-[(phenylsulfanyl)methyl]-1H1,2,3-triazole 15

White powder; yield (85\%) m.p. 97.7-98.7 ${ }^{\circ} \mathrm{C}$; FTIR $v_{\max }$ : $3152(=\mathrm{C}-\mathrm{H}, \mathrm{Tz}), 3075\left(=\mathrm{C}-\mathrm{H}_{\mathrm{Ar}}\right) 1581(\mathrm{C}=\mathrm{C}, \mathrm{Tz}), 1479$ $(\mathrm{N}=\mathrm{N}) \mathrm{cm}^{1} ;{ }^{1} \mathrm{H}$ NMR $\left(600 \mathrm{MHz}, \mathrm{CDCl}_{3}\right) \delta 7.46(\mathrm{t}, J=$ $10.2 \mathrm{~Hz}, \mathrm{H}-9), 7.27-7.13$ (m, 6H, $2 \mathrm{H}-13,2 \mathrm{H}-14, \mathrm{H}-5,1 \mathrm{H}-$ 15), 7.01 (d, $J=8.3 \mathrm{~Hz}, 2 \mathrm{H}, 2 \mathrm{H}-8$ ), 5.38 (s, $2 \mathrm{H}, \mathrm{H}-6), 4.20$ $(\mathrm{d}, J=12.6 \mathrm{~Hz}, 2 \mathrm{H}, \mathrm{H}-11) ;{ }^{13} \mathrm{C} \mathrm{NMR}\left(151 \mathrm{MHz}, \mathrm{CDCl}_{3}\right) \delta$ 145.60 (C-4), 133.57 (C-7), 132.22 (C-9), 126.67 (C-10), 122.41 (C-5), 53.39 (C-6), 28.88 (C-11); HRMS (APCI) $m / z:[\mathrm{M}+\mathrm{H}]^{+} 360.0153$ (calc. for $\mathrm{C}_{16} \mathrm{H}_{15} \mathrm{BrN}_{3} \mathrm{~S}: 360.0170$ ); purity (HPLC): $100 \%$.

\section{4-butyl-1-[(4-nitrophenyl)methyl]-1H-1,2,3-triazole 16}

Off yellow powder; yield (84\%) m.p. $63.1-65.9^{\circ} \mathrm{C}$; FTIR $v_{\max }: 2925 \quad(=\mathrm{C}-\mathrm{H}, \quad \mathrm{Tz}), \quad 1607 \quad(\mathrm{C}=\mathrm{C}, \quad \mathrm{Tz}), 1514$ $(\mathrm{N}=\mathrm{N}) \mathrm{cm}^{-1} ;{ }^{1} \mathrm{H}$ NMR $\left(600 \mathrm{MHz}, \mathrm{CDCl}_{3}\right) \delta 8.19(\mathrm{~d}, J=$ $7.6 \mathrm{~Hz}, 2 \mathrm{H}, \mathrm{H}-9), 7.34$ (d, $J=7.8 \mathrm{~Hz}, 2 \mathrm{H}, \mathrm{H}-8), 5.67$ (s, $2 \mathrm{H}$, H-6), 2.68 (s, 2H, H-11), 1.71 (s, $2 \mathrm{H}, 2 \mathrm{H}, \mathrm{H}-12$ ), 1.38 (s, 2H, H-13), 0.97-0.84 (m, 3H, H-14); ${ }^{13} \mathrm{C}$ NMR (151 MHz, $\left.\mathrm{CDCl}_{3}\right) \delta 147.96$ (C-10), 128.51 (C-9), 124.17 (C-8), 25.22 (C-11), 22.24 (C-12, -13), 13.75 (C-14); HRMS (APCI)

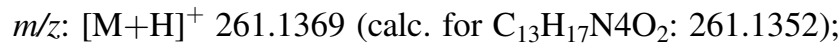
purity (HPLC): $97 \%$.

\section{4-hexyl-1-[(4-nitrophenyl)methyl]-1H-1,2,3-triazole 17}

Off yellow powder; yield (78\%); m.p. 75.5-75.6 ${ }^{\circ} \mathrm{C}$; FTIR $v_{\max }: 2905(\mathrm{C}-\mathrm{H}), 1606(\mathrm{C}=\mathrm{C}) \mathrm{cm}^{-1}$; ${ }^{1} \mathrm{H} \mathrm{NMR}(600 \mathrm{MHz}$, $\left.\mathrm{CDCl}_{3}\right) \delta 8.19(\mathrm{~d}, J=7.8 \mathrm{~Hz}, 2 \mathrm{H}, \mathrm{H}-9), 7.34(\mathrm{~d}, J=8.1 \mathrm{~Hz}$, $2 \mathrm{H}, \mathrm{H}-8), 5.65$ (s, 2H, H-6), 2.64 (d, $J=42.6 \mathrm{~Hz}, 2 \mathrm{H}$, $\mathrm{H}-11), 1.71$ (s, 2H, H-12), 1.45-1.08 (m, 6H, H-13, -14, -15), 0.97-0.68 (m, 3H, H-16); ${ }^{13} \mathrm{C}$ NMR (151 MHz, $\left.\mathrm{CDCl}_{3}\right) \delta 147.96$ (C-10), 128.47 (C-9), 124.17 (C-8), 31.46 (C-14) 28.84 (C-12, -13), 22.47 (C-15), 13.99 (C-16); HRMS (APCI) $m / z:[\mathrm{M}+\mathrm{H}]^{+} 289.1667$ (calc. for $\mathrm{C}_{15} \mathrm{H}_{21} \mathrm{~N}_{4} \mathrm{O}_{2}$ : 289.1665); purity (HPLC): $95 \%$.

\section{1-[(4-nitrophenyl)methyl]-4-octyl-1H-1,2,3-triazole 18}

Off yellow powder; yield (75\%); m.p. $76.1-76.8^{\circ} \mathrm{C}$; FTIR $v_{\max }: 3060\left(\mathrm{C}_{\mathrm{Ar}}-\mathrm{H}\right), 2919(=\mathrm{C}-\mathrm{H}), 1606(\mathrm{C}=\mathrm{C}), 1463$ $(\mathrm{N}=\mathrm{N}) \mathrm{cm}^{-1} ;{ }^{1} \mathrm{H}$ NMR $\left(600 \mathrm{MHz}, \mathrm{CDCl}_{3}\right) \delta 8.18(\mathrm{t}, J=$ 
$7.9 \mathrm{~Hz}, 2 \mathrm{H}, \mathrm{H}-9), 7.39-7.25$ (m, 2H, H-8), 5.60 (s, 2H, C-6), 2.68 (s, 2H, H-11), 1.63 (s, 2H, H-12), 1.39-1.07 (m, $10 \mathrm{H}, \mathrm{H}-13,-14,-15,-16,-17), 0.83$ (t, $J=6.9 \mathrm{~Hz}, 3 \mathrm{H}, \mathrm{H}-$ 18); ${ }^{13} \mathrm{C}$ NMR (151 MHz, $\left.\mathrm{CDCl}_{3}\right) \delta 147.92(\mathrm{C}-10), 142.03$ (C-7), 128.40 (C-8), 124.20 (C-9), 53.03 (C-6), 31.77 (C16), 25.63 (C-11), 22.59 (C-17), 14.04 (C-18); HRMS (APCI) $m / z:[\mathrm{M}+\mathrm{H}]^{+} 317.1990$ (calc. for $\mathrm{C}_{17} \mathrm{H}_{25} \mathrm{~N}_{4} \mathrm{O}_{2}$ : 317.1978); purity (HPLC): $82 \%$.

\section{4-decyl-1-[(4-nitrophenyl)methyl]-1H-1,2,3-triazole 19}

White powder; yield $(76 \%)$ m.p. $85.9-86.3^{\circ} \mathrm{C}$; FTIR $v_{\max }$ : $2913(=\mathrm{C}-\mathrm{H}), 2848,1522(\mathrm{C}=\mathrm{C}), 1470(\mathrm{~N}=\mathrm{N}) \mathrm{cm}^{-1} ;{ }^{1} \mathrm{H}$ NMR $\left(600 \mathrm{MHz}, \mathrm{CDCl}_{3}\right) \delta 8.19(\mathrm{~d}, J=8.3 \mathrm{~Hz}, 2 \mathrm{H}, \mathrm{H}-9)$, $7.35(\mathrm{~d}, J=8.4 \mathrm{~Hz}, 2 \mathrm{H}, \mathrm{H}-8), 5.60$ (s, 2H, H-6), 2.68 (s, $2 \mathrm{H}, \mathrm{H}-11), 1.64$ (s, 2H, H-12), 1.37-1.03 (m, 14H, H-13, $-14,-15,-16,-17,-18,-19), 0.84(\mathrm{t}, J=7.0 \mathrm{~Hz}, 3 \mathrm{H}, \mathrm{H}-20)$; ${ }^{13} \mathrm{C}$ NMR (151 MHz, CDCl3) $\delta 147.93$ (C-10), 142.01 (C7), 128.40 (C-9), 124.20 (C-8), 53.14 (C-6), 25.64 (C-11), 14.06 (C-20); HRMS (APCI) $m / z:[\mathrm{M}+\mathrm{H}]^{+} 345.2290$ (calc. for $\mathrm{C}_{19} \mathrm{H}_{29} \mathrm{~N}_{4} \mathrm{O}_{2}: 345.2291$ ); purity (HPLC): $93 \%$.

\section{1-[(4-nitrophenyl)methyl]-4-[(phenylsulfanyl)methyl]-1H- 1,2,3-triazole 20}

Yellow crystal; yield (80\%); m.p. 74.1-75.0 ${ }^{\circ} \mathrm{C}$; FTIR $v_{\max }$ : $1516(\mathrm{C}=\mathrm{C}), 1419(\mathrm{~N}=\mathrm{N}) \mathrm{cm}^{-1} ;{ }^{1} \mathrm{H}$ NMR $(600 \mathrm{MHz}$, $\left.\mathrm{CDCl}_{3}\right) \delta 8.16(\mathrm{~d}, J=8.6 \mathrm{~Hz}, 2 \mathrm{H}, \mathrm{H}-9), 7.35-7.09(\mathrm{~m}, 8 \mathrm{H}$, $2 \mathrm{H}-8,-13,-14,-15), 5.54$ (s, 2H, H-6), 4.21 (s, 2H, H-11); ${ }^{13} \mathrm{C}$ NMR $\left(151 \mathrm{MHz}, \mathrm{CDCl}_{3}\right) \delta 147.94(\mathrm{C}-10), 141.66(\mathrm{C}-$ 7), 135.03 (C-12), 124.21 (C-9), 53.02 (H-6), 28.71 (C-11); HRMS (APCI) $\mathrm{m} / \mathrm{z}:[\mathrm{M}+\mathrm{H}]^{+} 327.0927$ (calc. for $\left.\mathrm{C}_{16} \mathrm{H}_{15} \mathrm{~N}_{4} \mathrm{O}_{2} \mathrm{~S}: 327.0916\right)$; purity (HPLC): $96 \%$.

\section{4-butyl-1-\{[4-(trifluoromethyl)phenyl]methyl\}-1H-1,2,3- triazole 21}

Fine white powder; yield (70\%); m.p. $62.7-66.4^{\circ} \mathrm{C}$; FTIR $v_{\max }$ : $2915(=\mathrm{C}-\mathrm{H}), 1321(\mathrm{C}-\mathrm{F}) \mathrm{cm}^{-1}$; ${ }^{1} \mathrm{H}$ NMR $(600 \mathrm{MHz}$, $\left.\mathrm{CDCl}_{3}\right) \delta 7.64(\mathrm{~d}, J=7.8 \mathrm{~Hz}, 2 \mathrm{H}, \mathrm{H}-9), 7.36(\mathrm{~d}, J=7.9 \mathrm{~Hz}$, 2H, H-8), 5.60 (s, 2H, H-6), 2.72 (s, 2H, H-11), 1.69 (s, 2H, $\mathrm{H}-12), 1.40$ (d, $J=5.4 \mathrm{~Hz}, 2 \mathrm{H}, 2 \mathrm{H}, \mathrm{H}-13), 0.94$ (t, $J=7.1 \mathrm{~Hz}$, $3 \mathrm{H}, \mathrm{H}-14) ;{ }^{13} \mathrm{C}$ NMR (151 MHz, $\left.\mathrm{CDCl}_{3}\right) \delta 138.90$ (C-7), 131.02 (m, C-10), 128.13 (C-8), 126.05 (C-9), 123.80 (d, $J=$ $\left.272.3 \mathrm{~Hz}, \mathrm{C}-10^{\prime}\right), 32.17-30.49$ (C-12), 25.41 (C-11), 22.34 (C-13), 13.83 (C-14); HRMS (APCI) $m / z:[\mathrm{M}+\mathrm{H}]^{+} 284.1344$ (calc. for $\mathrm{C}_{14} \mathrm{H}_{17} \mathrm{~F}_{3} \mathrm{~N}_{3}$ : 284.1375); purity (HPLC): $96 \%$.

\section{4-hexyl-1-\{[4-(trifluoromethyl)phenyl]methyl\}-1H-1,2,3- triazole 22}

White powder; yield (81\%); m.p. $68.5-69.8^{\circ} \mathrm{C}$; FTIR $v_{\max }$ : $2917(=\mathrm{C}-\mathrm{H}), 1325(\mathrm{C}-\mathrm{F}) \mathrm{cm}^{-1} ;{ }^{1} \mathrm{H}$ NMR $(600 \mathrm{MHz}$,
$\left.\mathrm{CDCl}_{3}\right) \delta 7.65(\mathrm{~d}, J=7.5 \mathrm{~Hz}, 2 \mathrm{H}, \mathrm{H}-9), 7.35(\mathrm{~d}, J=7.6 \mathrm{~Hz}$, $2 \mathrm{H}, \mathrm{H}-8), 5.63$ (s, 2H, H-6), 2.90-2.50 (m, $2 \mathrm{H}, 2 \mathrm{H}, \mathrm{H}-11$ ), $1.77(\mathrm{~d}, J=39.8 \mathrm{~Hz}, 2 \mathrm{H}, 2 \mathrm{H}, \mathrm{H}-12), 1.47-1.22(\mathrm{~m}, 6 \mathrm{H}, \mathrm{H}-$ $13,-14,-15), 0.90(\mathrm{~d}, J=6.0 \mathrm{~Hz}, 3 \mathrm{H}, \mathrm{H}-16) ;{ }^{13} \mathrm{C}$ NMR $\left(151 \mathrm{MHz}, \mathrm{CDCl}_{3}\right) \delta 138.78(\mathrm{C}-7), 130.95$ (d, $J=32.4 \mathrm{~Hz}$, C-10), 128.18 (C-8), 126.04 (C-9), 123.79 (d, $J=272.2 \mathrm{~Hz}$, C-10'), 28.57 (C-12), 25.74 (C-11), 14.07 (C-16); HRMS (APCI) $m / z:[\mathrm{M}+\mathrm{H}]^{+} 312.1654$ (calc. for $\mathrm{C}_{16} \mathrm{H}_{21} \mathrm{~F}_{3} \mathrm{~N}_{3}$ : 312.1688); purity (HPLC): $95 \%$.

\section{4-octyl-1-\{[4-(trifluoromethyl)phenyl]methyl\}-1H-1,2,3- triazole 23}

White powder; yield (70\%); m.p. 81.9-82. ${ }^{\circ} \mathrm{C}$; FTIR $v_{\max }$ : $3112(=\mathrm{C}-\mathrm{H}, \mathrm{Tz}), 3058\left(=\mathrm{C}-\mathrm{H}_{\mathrm{Ar}}\right), 1618(\mathrm{C}=\mathrm{C}, \mathrm{Tz}), 1327$ $(\mathrm{C}-\mathrm{F}) \mathrm{cm}^{-1}$; ${ }^{1} \mathrm{H}$ NMR $\left(600 \mathrm{MHz}, \mathrm{CDCl}_{3}\right) \delta 7.63(\mathrm{~d}, J=$ $7.7 \mathrm{~Hz}, 2 \mathrm{H}, \mathrm{H}-9), 7.34$ (d, $J=7.7 \mathrm{~Hz}, 2 \mathrm{H}, \mathrm{H}-8), 5.60$ (s, 2H, H-6), 2.70 (s, 2H, H-11), 1.72 (d, $J=35.8 \mathrm{~Hz}, 2 \mathrm{H}, \mathrm{H}-12$ ), $1.42-1.13$ (m, 10H, H-13, -14, -15, -16, -17), 0.87 (t, $J=$ $7.0 \mathrm{~Hz}, 3 \mathrm{H}, \mathrm{H}-18) ;{ }^{13} \mathrm{C}$ NMR $\left(151 \mathrm{MHz}, \mathrm{CDCl}_{3}\right) \delta 138.89$ (C-7), 130.94 (d, $J=32.6 \mathrm{~Hz}, \mathrm{C}-10), 128.14$ (C-8), 126.05 (C-9), 123.80 (d, $\left.J=272.2 \mathrm{~Hz}, \mathrm{C}-10^{\prime}\right), 25.76$ (C-11), 14.12 (H-18); HRMS (APCI) $m / z:[\mathrm{M}+\mathrm{H}]^{+} 340.1987$ (calc. for $\mathrm{C}_{18} \mathrm{H}_{25} \mathrm{~F}_{3} \mathrm{~N}_{3}$ : 340.2001); purity (HPLC): $97 \%$.

\section{4-decyl-1-\{[4-(trifluoromethyl)phenyl]methyl\}-1H-1,2,3- triazole 24}

Fine white powder; yield (65\%), m.p. $78.6-79.2^{\circ} \mathrm{C}$; FTIR $v_{\max }: 3112(=\mathrm{C}-\mathrm{H}, \mathrm{Tz}), 3059\left(=\mathrm{C}-\mathrm{H}_{\mathrm{Ar}}\right), 1621(\mathrm{C}=\mathrm{C}, \mathrm{Tz})$, $1468(\mathrm{~N}=\mathrm{N}), 1331(\mathrm{C}-\mathrm{F}) \mathrm{cm}^{-1}$; ${ }^{1} \mathrm{H}$ NMR $(600 \mathrm{MHz}$, $\left.\mathrm{CDCl}_{3}\right) \delta 7.65(\mathrm{~d}, J=7.5 \mathrm{~Hz}, 2 \mathrm{H}, \mathrm{H}-9), 7.36(\mathrm{~d}, J=7.3 \mathrm{~Hz}$, 2H, H-8), 5.62 (s, 2H, H-6), 2.72 (s, 2H, H-11), 1.75 (d, $J=34.3 \mathrm{~Hz}, 2 \mathrm{H}, \mathrm{H}-12), 0.89$ (t, $J=7.0 \mathrm{~Hz}, 3 \mathrm{H}, \mathrm{H}-20) ;{ }^{13} \mathrm{C}$ NMR $\left(151 \mathrm{MHz}, \mathrm{CDCl}_{3}\right) \delta 138.87(\mathrm{C}-7), 130.95$ (d, $J=$ $32.0 \mathrm{~Hz}, \mathrm{C}-10), 128.16$ (C-8), 126.05 (C-9), 123.79 (d, $J=$ $\left.272.3 \mathrm{~Hz}, \mathrm{C}-10^{\prime}\right), 25.45$ (C-11), 14.14 (C-20); HRMS (APCI) $m / z:[\mathrm{M}+\mathrm{H}]^{+} 368.2305$ (calc. for $\mathrm{C}_{20} \mathrm{H}_{29} \mathrm{~F}_{3} \mathrm{~N}_{3}$ : 368.2314); purity (HPLC): $90 \%$.

\section{4-[(phenylsulfanyl)methyl]-1-\{[4-(trifluoromethyl)phenyl] methyl\}-1H-1,2,3-triazole 25}

Off-white powder; yield (65\%); m.p. $123.7-126.9^{\circ} \mathrm{C}$; FTIR $v_{\max }: 3122(=\mathrm{C}-\mathrm{H}, \mathrm{Tz}), 3081\left(=\mathrm{C}-\mathrm{H}_{\mathrm{Ar}}\right), 1619(\mathrm{C}=\mathrm{C}, \mathrm{Tz})$, $1424(\mathrm{~N}=\mathrm{N}), 1326(\mathrm{C}-\mathrm{F}) \mathrm{cm}^{1} ;{ }^{1} \mathrm{H}$ NMR $\left(600 \mathrm{MHz}, \mathrm{CDCl}_{3}\right)$ $\delta 7.60(\mathrm{~d}, J=8.1 \mathrm{~Hz}, 2 \mathrm{H}, \mathrm{H}-9), 7.32-7.14(\mathrm{~m}, 7 \mathrm{H}, \mathrm{H}-8$, $-13,-14,-15), 5.52$ (s, 2H, H-6), 4.22 (s, 2H, H-11); ${ }^{13} \mathrm{C}$ NMR $\left(151 \mathrm{MHz}, \mathrm{CDCl}_{3}\right) \delta 138.55(\mathrm{C}-7), 135.08(\mathrm{C}-12)$, 130.99 (d, $J=32.6 \mathrm{~Hz}, \mathrm{C}-10), 123.78(\mathrm{~d}, J=272.3 \mathrm{~Hz}$, C-10'), 53.52 (C-6), 28.88 (C-11); HRMS (APCI) $\mathrm{m} / \mathrm{z}$ : $[\mathrm{M}+\mathrm{H}]^{+} 350.0938$ (calc. for $\mathrm{C}_{17} \mathrm{H}_{15} \mathrm{~F}_{3} \mathrm{~N}_{3} \mathrm{~S}$ : 350.0939); purity (HPLC): $92 \%$. 


\section{In vitro biological evaluation}

\section{Antimycobacterial activity assessment}

The minimum inhibitory concentration (MIC) was determined using the standard broth micro dilution method, where a $10 \mathrm{ml}$ culture of $M t b$ pMSp12::GFP (Abrahams et al. 2012; Collins and Franzblau 1997; Collins et al. 1998) is grown to an optical density (OD600) of 0.6-0.7. Cultures are diluted, prior to inoculation of assays, as follows:

(1) 1:100 in Gaste-Fe (glycerol-alanine-salts) medium pH 6.6, supplemented with $0.05 \%$ Tween-80 and $1 \%$ glycerol (De Voss et al. 2000; Franzblau et al. 2012).

(2) $1: 500$ in $7 \mathrm{H} 9$ supplemented with $10 \%$ albumin dextrose catalase supplement (ADC), $0.4 \%$ glucose and $0.05 \%$ Tween- 80 (De Voss et al. 2000; Franzblau et al. 2012).

The compounds to be tested are reconstituted in DMSO. Twofold serial dilutions of the test compound are prepared across a 96-well microtiter plate, after which $50 \mu \mathrm{l}$ of the diluted $M t b$ cultures are added to each well in the serial dilution. The plate layout is a modification of the method previously described (Ollinger et al. 2013). Assay controls used are a minimum growth control (RIF at $2 \times$ MIC), and a maximum growth control (5\% DMSO). The microtiter plates are sealed in a secondary container and incubated at $37^{\circ} \mathrm{C}$ with $5 \% \mathrm{CO} 2$ and humidification. Relative fluorescence (excitation $485 \mathrm{nM}$; emission $520 \mathrm{nM}$ ) is measured using a plate reader (FLUOstar OPTIMA, BMG LABTECH) at day 7 and day 14. The raw fluorescence data are archived and analysed using the CDD Vault from Collaborative Drug Discovery, in which data are normalised to the minimum and maximum inhibition controls to generate a dose response curve (\% inhibition), using the Levenberg-Marquardt damped least-squares method, from which the MIC 90 is calculated (Burlingame, CA, www.collaborativedrug.com). The lowest concentration of drug that inhibits growth of more than $90 \%$ of the bacterial population is considered to be the MIC90.

\section{Cytotoxicity assay}

Human embryonic kidney (HEK-293) cells (ATCC ${ }^{\circ} \mathrm{CRL}^{-}$ $1573^{\mathrm{TM}}$ ) were cultured in Hyclone Dulbecco's modified Eagle's medium with high glucose supplemented with $10 \%$ foetal bovine serum and $1 \%$ L-glutamine, Penicillin-Streptomycin, Amphotericin B and non-essential amino acids. Cell lines are maintained in a humidified atmosphere at $37{ }^{\circ} \mathrm{C}$ and $5 \% \mathrm{CO}$. For the MTT assay, 96well plates were prepared with $200 \mu \mathrm{l}$ of cell suspension
(75,000 cells $/ \mathrm{ml})$ and incubated for $24 \mathrm{~h}$. The cells were then treated with: (1) $100 \mu \mathrm{l}$ of emetine dihydrochloride solution diluted with growth medium to the necessary concentrations (positive control); (2) $80 \mu \mathrm{l}$ of growth medium and $20 \mu \mathrm{l}$ of solvent (negative control to compensate for possible solvent effects); (3) $80 \mu \mathrm{l}$ of growth medium and $20 \mu \mathrm{l}$ of experimental compound solutions. Blanks contained growth medium without cells. The treated plates were incubated for $48 \mathrm{~h}$.

To initiate the MTT assay, $20 \mu \mathrm{l}$ of sterile-filtered MTT solution $(1 \mathrm{mg} / \mathrm{ml}$ in PBS) was added and the plates incubated for $4 \mathrm{~h}$. The growth medium-MTT mixture was then aspirated and $100 \mu \mathrm{l}$ of 2-propanol was added to dissolve purple formazan crystals. Absorbance was measured at 560 and $650 \mathrm{~nm}$ using the Thermofisher Scientific GO Multiscan plate reader. Due to light sensitivity of MTT reagent, the assay was performed in the dark. Thus, the plates were covered with aluminium foil and the contents gently mixed for $5 \mathrm{~min}$ at room temperature. Data analysis was performed for each biological replicate using SkanIt 4.0 Research Edition software. Background absorbance $(650 \mathrm{~nm})$ was subtracted from absorbance values $(560 \mathrm{~nm})$, the mean absorbance calculated and the percentage cell viability was determined by the following equation:

$$
\begin{aligned}
\text { Cell viability } \%= & (\Delta \text { Abs sample }-\Delta \text { Abs blank }) / \\
& (\Delta \text { Abs neg control }-\Delta \text { Abs blank }) \times 100
\end{aligned}
$$

The $\mathrm{IC}_{50}$ and $Z$-score were determined for each compound's biological replicate using the SkanIt 4.0 software and four-parameter logistic (sigmoidal) regression. For the final $\mathrm{IC}_{50}$ of each compound, the mean $\mathrm{IC}_{50}$ of the biological replicates were calculated.

\section{Results and discussion}

\section{Chemistry}

Click chemistry is a term that initially described reactions giving high yields and selectivity products by carbon-hetero bond formation. The word "click" refers to easily joining molecular building blocks, such as two pieces of a seat belt buckle. Of the reactions comprising the click universe, the gold standard is the Huisgen 1,3-dipolar cycloaddition of alkynes with azides to form 1,4-disubsituted-1,2,3-triazoles as depicted in Scheme 1. This copper(I)-catalyzed reaction is mild and very efficient, requiring no protecting groups and no purification in the majority of cases (Tornøe et al. 2002). The azide and alkyne functional groups are largely inert towards biological molecules and aqueous environments, allowing the use of 
the Huisgen 1,3-dipolar cycloaddition in target-guided synthesis (Manetsch et al. 2004) and activity-based protein profiling (Speers et al. 2003). The resulting triazole scaffold has similarities to the ubiquitous amide moiety found in nature. However, unlike the amide bond, the triazole ring is rigid and not susceptible to cleavage. In addition, it is impossible to oxidize or reduce.

A series of BZT derivatives were easily synthesized in a single step using a literature reported method employing copper-catalyzed azide-alkyne 1,3-dipolar cycloaddition $(\mathrm{CuAAC})$ in a THF-MeOH-water (v/v/v, 1:1:1) mixture, an adaptation of the procedure by Shin et al. (2012) as depicted

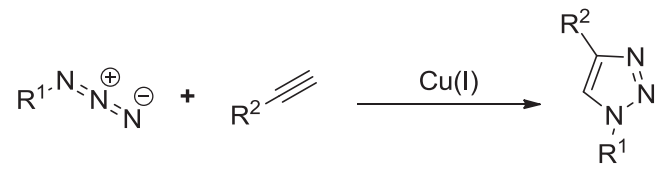

$$
\begin{aligned}
& \text { azide alkyne 1,2,3-triazole }
\end{aligned}
$$

Scheme 1 Schematic representation of copper(I)-catalyzed azide/ alkyne click reaction in Scheme 2. The reaction is an in situ process involving two major reactions, $\mathrm{S}_{\mathrm{N}} 2$ and $\mathrm{CuAAC}$. The reagents include benzyl bromide (electrophile source), $\mathrm{NaN}_{3}$ (nucleophile source), copper (II) sulfate (catalyst), sodium ascorbate (reducing agent), $\beta$-CD (phase transfer catalyst) and alkyne (nucleophile source). The nucleophilic substitution $\mathrm{S}_{\mathrm{N}} 2$ of the selected benzyl bromide with $\mathrm{NaN}_{3}$ results in a benzylazide intermediate by adopting the synthetic route described by Hodson and Peter (2010). Synchronously, the benzylazide intermediate undergoes a Huisgen copper alkyne-azide 1,3-dipolar cycloaddition "click" reaction with the alkyne over $24 \mathrm{~h}$ (Shin et al. 2012). The later reaction is catalyzed by copper (II) while $\beta$-CD serves as phase transfer catalyst between the lipophilic reagents (benzylazide and alkyne) and the aqueous phase, thus homogenizing the medium. It is noteworthy to indicate that the solvent combination (THF-MeOH- $\left.\mathrm{H}_{2} \mathrm{O} ; 1: 1: 1, \mathrm{v} / \mathrm{v} / \mathrm{v}\right)$ was identified as affording the best yields after several solvents, including DMSO, acetone, water, $\mathrm{MeOH}$ and $\mathrm{THF}$, were attempted. Another note of particular importance is the sequential

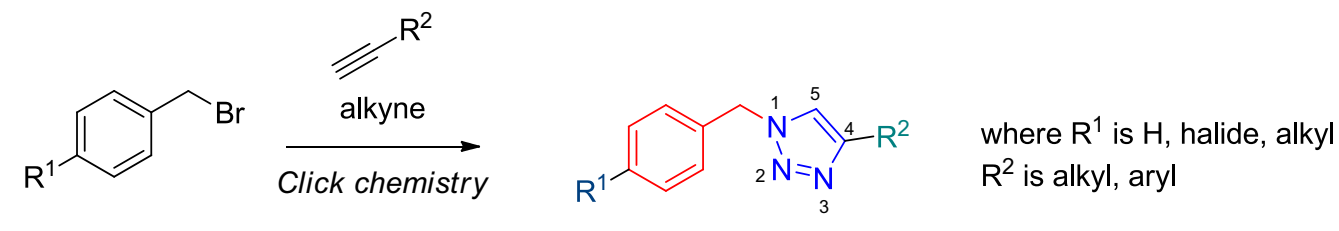

Benzyl bromide

1-Benzyl-1,2,3-triazoles, 1 - 25

\begin{tabular}{|c|c|c|c|c|c|}
\hline Compd. & $\mathbf{R}^{1}$ & $\mathbf{R}^{2}$ & Compd & $\mathbf{R}^{1}$ & $\mathbf{R}^{2}$ \\
\hline 1 & $\mathrm{H}$ & $n-\mathrm{C}_{4} \mathrm{H}_{9}$ & 14 & $"$ & $n-C$ \\
\hline 2 & $"$ & $n-\mathrm{C}_{6} \mathrm{H}_{13}$ & 15 & $"$ & \\
\hline $\begin{array}{l}3 \\
4\end{array}$ & " & $\begin{array}{l}n-C_{8} \vdash \\
n-C_{10}\end{array}$ & $\begin{array}{l}16 \\
17\end{array}$ & $\mathrm{NO}_{2}$ & \\
\hline 5 & $"$ & & 18 & $"$ & $n-\mathrm{C}_{8} \mathrm{H}_{17}$ \\
\hline 6 & $\mathrm{CH}_{3}$ & $n-\mathrm{C}_{4} \mathrm{H}_{9}$ & 19 & $"$ & \\
\hline 7 & $"$ & $n-\mathrm{C}_{6} \mathrm{H}_{13}$ & 20 & $"$ & \\
\hline $\begin{array}{l}8 \\
9\end{array}$ & " & $\begin{array}{l}n-\mathrm{C}_{8} \mathrm{H}_{17} \\
n-\mathrm{C}_{10} \mathrm{H}_{21}\end{array}$ & $\begin{array}{l}21 \\
22\end{array}$ & $\mathrm{CF}_{3}$ & $\begin{array}{c}n-\mathrm{C}_{4} \mathrm{H}_{9} \\
n-\mathrm{C}_{6} \mathrm{H}_{13}\end{array}$ \\
\hline 10 & $"$ & & 23 & $"$ & $n-\mathrm{C}_{8} \mathrm{H}_{17}$ \\
\hline 11 & $\mathrm{Br}$ & $n-\mathrm{C}_{4} \mathrm{H}_{9}$ & 24 & $"$ & $n-\mathrm{C}_{10} \mathrm{H}_{21}$ \\
\hline 12 & $"$ & $n-\mathrm{C}_{6} \mathrm{H}_{13}$ & 25 & $"$ & \\
\hline 13 & $"$ & $n-\mathrm{C}_{8} \mathrm{H}_{17}$ & & & \\
\hline
\end{tabular}

Scheme 2 Synthesis of benzyltriazole derivatives 
addition of reagents. The current sequence was adopted after several other sequences failed to deliver the target compounds. In those attempts, a quick formation of a black solution occurred, indicative of the oxidation of the copper (II) to copper (I) due to fast addition of reagent, incorrect adding sequence or inappropriate thermal conditions. This reaction could be reverted if detected soon enough with the addition of a portion of the reducing agent, sodium ascorbate. The target compounds 1-25 were obtained as 1,4disubstituted-1,2,3-triazoles and were isolated in moderate to excellent yields (60-95\%) after purification by crystallization in hexane.

Reagents and conditions: alkyne (1.2 eq.), THF/MeOH/ $\mathrm{H}_{2} \mathrm{O}$ (1/1/1; v/v/v), $\beta$-CD (0.02 eq.), NaAsc (0.2 eq.), $\mathrm{NaN}_{3}$ (1.2 eq.), $\mathrm{CuSO}_{4} \cdot 5 \mathrm{H}_{2} \mathrm{O}$ (0.2 eq.), rt, $10-24 \mathrm{~h}$.

In order to characterize the novel compounds, analytical instruments and techniques were used as described in the general procedures. Compounds with similar substitutions showed similar signal patterns on their NMR and IR spectra. The ${ }^{1} \mathrm{H}$ NMR spectra of all title compounds were thoroughly examined for characteristic peaks, evidence of the phenyl and triazole rings.

For compounds 1-5, which are unsubstituted on para position of the phenyl ring, peaks of the five aromatic protons and that of the triazolyl proton (H-5) overlapped as a multiplet in the 7.4-7.15 ppm region. In addition, the singlet $c a .5 .40 \mathrm{ppm}$ was assigned to the two methylene protons of benzyl moiety, while the terminal $\mathrm{CH}_{3}$ in all the aliphatic chains (1-4) appeared as a multiplet or a triplet with ${ }^{3} J \sim 0.9 \mathrm{~Hz}$ as a result of the coupling with the two protons of the neighbouring/preceding methylene group. In compound 5, an additional singlet $c a .4 .19 \mathrm{ppm}$ was attributed to the protons (H-11) of the methylene group linked to the thiophenyl moiety, while further peaks of the aromatic protons of that moiety were found in the abovementioned chemical shift range. In the ${ }^{1} \mathrm{H}$ NMR spectra of compounds 6-10 ( $p$-methyl substituted), peaks of the aromatic protons appeared as a multiplet in the region of 7.13-7.08 ppm. Methylene protons (H-6) of benzyl moiety gave a singlet around $5.4 \mathrm{ppm}$, while protons of the methylene linked to the thiophenyl group appeared as a singlet at $4.2 \mathrm{ppm}$ in compound 10. The $p$-methyl protons $\left(\mathrm{H}-10^{\prime}\right)$ were assigned to the singlet around $2.3 \mathrm{ppm}$, while the alkyl chain terminal $\mathrm{CH}_{3}$ gave a triplet in the 0.9-0.8 ppm region for compounds 6-9. All abovementioned characteristic peaks were also found in the ${ }^{1} \mathrm{H}$ spectra of 11-25.

In ${ }^{13} \mathrm{C}$ spectra, the phenyl carbons gave peaks in the 130-125 ppm region, while the resonance of triazolyl carbons (C-4 and C-5) resulted in singlets in the 128-125 ppm region.

The FTIR-spectra of all compounds were also inspected for the presence of characteristic absorptions, allowing for the identification of functional groups. Evidence of the triazolyl moiety was confirmed with bends of $\mathrm{C}=\mathrm{C}$ and $\mathrm{N}=\mathrm{N}$ bonds $c a .1600$ and $1450 \mathrm{~cm}^{-1}$, respectively, while vibration of the $\mathrm{H}-\mathrm{C}=$ bond gave a stretch at around $3100 \mathrm{~cm}^{-1}$. Of note, vibrations of $\mathrm{C}-\mathrm{F}$ bonds of the trifluoromethyl substituent resulted in rockings around $1300 \mathrm{~cm}^{-1}$ in the spectra of compounds 21-25.

\section{Physiochemical properties}

Transmembrane transport gives a good indication of the biological properties, such as oral bioavailability and cellular uptake, and pharmacological activity of a compound (Gombar and Enslein 1996). An ideal drug must possess well-equipoised hydrophilic/lipophilic properties, so as to efficiently permeate biological membranes and be absorbed into the systemic circulation (Lipinski et al. 1997). The $n$ octanol/water partition coefficient $(\operatorname{cog} P)$ is a key parameter used in the measurement of the hydrophilicity and lipophilicity of a chemical, allowing one to predict the transport characteristics of a substance across biological membranes through passive diffusion (Gombar and Enslein 1996). The cLogP values between 1 and 5 are often targeted, with values between 1 and 3 being ideal (Lipinski et al. 1997). Thus, the lipophilic substituent $\mathrm{R}^{2}$ (n-alkyl or methylenediphenyl) side chains were anchored to the triazole ring to assess the influence of lipophilicity on the pharmacological effect of the compounds.

The substituent $\mathrm{R}^{1}\left(\mathrm{H}, \mathrm{CH}_{3}, \mathrm{Br}, \mathrm{NO}_{2}\right.$ and $\left.\mathrm{CF}_{3}\right)$, on the other hand, were introduced on the phenyl ring to judge the impact that electronic effects may have on the antimycobacterial activity.

Most BZT derivatives, with the exception of long $n$-alkyl side chain $8,9,13,14,19,23$ and 24 , showed good druglike properties with cLogP values within the targeted range. However, these above-mentioned threshold $\operatorname{cLogP}$ value compounds may still be active because biological activity is dependent on many parameters apart from physicochemical properties (Pop et al. 2004). This is better illustrated with the most recent drugs introduced in the market, bedaquiline (cLogP 7.25) and delamanid (cLogP 6.14), which have cLogP values above the safe threshold of 5 (Machado et al. 2018).

\section{Biological activities}

For a novel drug to advance to human use, a chain of preclinical studies, including in vitro assays and in vivo testing, need to be concluded.

The in vitro mycobacterial growth inhibitory potential of the synthesized compounds was assessed using the standard microplate green fluorescent protein (GFP) assay against Mtb H37Rv, using a reporter mutant constitutively 
Table 1 In vitro biological activities of synthesized benzyltriazoles

\begin{tabular}{|c|c|c|c|c|c|c|c|}
\hline \multirow[b]{2}{*}{ Cpd } & \multirow[b]{2}{*}{$\begin{array}{l}\text { Chain } \\
\text { length }(\mathrm{C})\end{array}$} & \multirow[b]{2}{*}{$\operatorname{cLog} \mathrm{P}^{\mathrm{a}}$} & \multicolumn{3}{|c|}{ Antimycobacterial activity $(\mu \mathrm{M})$} & \multirow{2}{*}{$\begin{array}{l}\text { Cytotoxicity, } \\
\mathrm{IC}_{50}(\mu \mathrm{M})^{\mathrm{d}} \\
\text { HEK-293 }\end{array}$} & \multirow{2}{*}{$\begin{array}{l}\text { Selectivity } \\
\text { index } \\
\mathrm{SI}^{\mathrm{e}}\end{array}$} \\
\hline & & & $\begin{array}{l}\mathrm{MIC}_{90} \\
\text { [GAST/Fe] }^{\mathrm{b}}\end{array}$ & $\begin{array}{l}\mathrm{MIC}_{99} \\
{[\mathrm{GAST} / \mathrm{Fe}]^{\mathrm{b}}}\end{array}$ & $\begin{array}{l}\mathrm{MIC}_{90} \\
\text { [7H9/ADC] }^{\mathrm{c}}\end{array}$ & & \\
\hline 1 & 4 & 3.5 & 7.76 & 7.7 & 62.5 & $>100$ & $>13$ \\
\hline 2 & 6 & 4.4 & 29.6 & 32.5 & 121.9 & $>100$ & $>3$ \\
\hline 3 & 8 & 5.2 & $>125$ & $>125$ & $>125$ & & \\
\hline 4 & 10 & 6.1 & $>125$ & $>125$ & $>125$ & & \\
\hline 5 & & 3.9 & 20 & 38.3 & $>125$ & $>100$ & $>5$ \\
\hline 6 & 4 & 4.0 & 19.6 & $>125$ & $>125$ & $>100$ & $>5$ \\
\hline 7 & 6 & 4.9 & 10.2 & 12.4 & $>125$ & $>100$ & $>10$ \\
\hline 8 & 8 & 5.8 & 29.9 & 31.2 & $>125$ & $>100$ & $>3$ \\
\hline 9 & 10 & 6.7 & $>125$ & $>125$ & $>125$ & & \\
\hline 10 & & 4.4 & 10.3 & 17.3 & $>125$ & $>100$ & $>10$ \\
\hline 11 & 4 & 4.2 & 2.78 & 3.82 & 58.5 & $>100$ & $>36$ \\
\hline 12 & 6 & 5.0 & nd & nd & 71.6 & $>100$ & \\
\hline 13 & 8 & 6.1 & 35.7 & 40.1 & $>125$ & $>100$ & $>3$ \\
\hline 14 & 10 & 6.9 & $>125$ & $>125$ & $>125$ & & \\
\hline 15 & & 4.6 & $>125$ & $>125$ & $>125$ & & \\
\hline 16 & 4 & 3.4 & 64.8 & $>125$ & $>125$ & $>100$ & $>2$ \\
\hline 17 & 6 & 4.3 & 66.6 & $>125$ & 66.12 & $>100$ & $>2$ \\
\hline 18 & 8 & 5.2 & $>125$ & $>125$ & $>125$ & & \\
\hline 19 & 10 & 6.1 & $>125$ & $>125$ & $>125$ & & \\
\hline 20 & & 3.8 & $>125$ & $>125$ & 82.03 & & \\
\hline 21 & 4 & 4.3 & 1.73 & 3.19 & 125 & $>100$ & $>58$ \\
\hline 22 & 6 & 5.2 & 86.3 & $>125$ & $>125$ & $>100$ & $>1$ \\
\hline 23 & 8 & 6.1 & $>125$ & $>125$ & $>125$ & & \\
\hline 24 & 10 & 7.1 & $>125$ & $>125$ & $>125$ & & \\
\hline 25 & & 4.8 & $>125$ & $>125$ & $>125$ & & \\
\hline INH & & -0.7 & 0.041 & 0.046 & 1.06 & & \\
\hline RIF & & 3.6 & 0.001 & 0.001 & 0.004 & & \\
\hline Em & & & & 3.906 & 125 & 0.2 & \\
\hline
\end{tabular}

${ }^{a}$ cLogP values calculated using MarvinSketch Version 17.28

${ }^{\mathrm{b}}$ Compounds screened in medium: protein-free GAST-Fe

${ }^{c}$ Compounds screened in medium: protein-rich 7H9 GLU ADC

${ }^{\mathrm{d}}$ Human embryonic kidney cell line from ECACC

${ }^{\text {e}}$ Selectivity index, $\mathrm{SI}=\mathrm{IC}_{50}$ HEK-293/ $\mathrm{MIC}_{90}$ H37Rv, Em (emetine) expressing GFP as described previously (Wilson et al. 2017) in two different media namely the Middlebrook 7H9 Broth base, supplemented with glucose (GLU) and enriched with ADC (albumin BSA-dextrose-catalyse), and GAST-Fe (glycerol-alanine-salts), BSA free (Soares de Melo et al. 2015). The assay was performed using the media to facilitate observation of culture-conditions mediating antimycobacterial activity.

The minimum concentrations of the compounds that inhibit the growth of 90 and $99 \%$ of mycobacteria, expressed as $\mathrm{MIC}_{90}$ and $\mathrm{MIC}_{99}$, respectively, are summarized in Table 1 alongside those of INH and RIF antitubercular standards.
Furthermore, HEK-293 cells were used to determine the cytotoxicity of the compounds alongside the cytotoxic drug emetine as reference.

Half of the synthesized compounds were active in GAST/Fe medium, while the overwhelming majority (72\%) were found to be inactive in 7H9/ADC medium. Even for potent compounds in both media, the activity was more pronounced in GAST/Fe than in 7H9/ADC. In particular, compound $\mathbf{1 1}$ was found with a 20-fold activity difference, while 1 was 8 times more active in the albumin-free medium instead. This could be due to the lack and presence of albumin in GAST/Fe and 7H9/ADC media, respectively. Indeed, serum albumins are the major soluble protein 
constituents of the circulatory system, accounting for $4 \%$ weight per volume $(\mathrm{w} / \mathrm{v})$. They have the ability to reversibly bind a large variety of exogenous compounds, including fatty acids, amino acids, drugs and pharmaceuticals (Dockal et al. 2000; Ran et al. 2007; Tian et al. 2003). Plasma protein binding $(\mathrm{PB})$ forms an integral part of distribution and bioavailability for numerous medications currently in clinical use; however, binding becomes problematic where it is extensive (Kandagal et al. 2006; Seetharamappa and Kamat 2004). The influence of PB on pharmacological effects is dependent on the biological setting. In vitro binding to albumin would reduce the amount of compound available to exert an effect on the mycobacterial cell and, in theory, reduce the observed MIC of compounds. In vivo, however, the impact of the albumin binding on MIC of compounds would depend on the extent and nature of the binding of the drug to albumin within the host.

The majority of compounds were inactive in 7H9/ADC, which may suggest an irreversible binding to BSA and the culprit would be the triazole, possibly binding to protein and other macromolecules (Massarotti et al. 2014) through its H-bonding acceptors ( $\mathrm{N}-2$ and $\mathrm{N}-3)$ or donor (H-5) sites (Fig. 1).

Albumin binding properties may also provide some insight into the possible pharmacokinetic behaviour of compounds. Binding and dissociation of compounds from plasma proteins is a dynamic process with only the unbound fraction of the drug available to exert an effect. In a clinical setting, the dosage of a drug is calculated to ensure that, at any point in time, sufficient free drug is available to have the required pharmacological effect. The same principle would apply to side effects or toxicity and thus, plasma PB becomes an important consideration, particularly in highly bound drugs.

In light of the inactivity of most compounds in $7 \mathrm{H} 9 /$ $\mathrm{ADC}$, the MIC values obtained in GAST-Fe medium were utilized for the purposes of determining structure activity relationships (SARs). Comparison of the activities in regard to the $n$-alkyl side chain length reveals that the short chain (4C and 6C) compounds were the most active compounds in GAST/Fe. However, 8 with its 8 carbons chain was also found to be active in the medium with $\mathrm{MIC}_{90} 29.9 \mu \mathrm{M}$. Overall, the long chains ( $8 \mathrm{C}$ and $10 \mathrm{C}$ ) derivatives were inactive regardless of the medium. The limited number of active compounds did not allow for a rigorous stand on the impact of the variation of chain in relation to resulting activity. However, the optimum chain, inducing maximum activity among active compounds in GAST/Fe, seems to be $4 \mathrm{C}$ as can be gleaned from the activities of $\mathbf{1}$ vs. $2 ; \mathbf{1 6}$ vs. 17; and 21 vs. 22.

Thiophenyl-containing derivatives presented a mixed activity profile. Derivatives $\mathbf{5}$ and 10, which bore phenyl and $p$-Me phenyl, respectively, were active with $\mathrm{MIC}_{90}$ values of 20 and $10.3 \mu \mathrm{M}$, respectively, in GAST/Fe. However, this activity was lost in 7H9/ADC medium. The remaining compounds, $\mathbf{1 5}, \mathbf{2 0}$ and $\mathbf{2 5}$ of this sub-series, were completely inactive irrespective of the assay medium.

Furthermore, consideration of the lipophilicity shows the most active compounds to be drug-likeable with $\operatorname{cLogP}$ values in the target range (1-5). Due to the lipophilic nature of the mycobacterium cell wall, one would have expected more activity from the more lipophilic compounds (Fan et al. 2018), but the opposite was observed in this study. Similarly, to the impact of the chain length, a realistic conclusion of the effect of lipophilicity on the activity could not be drawn due to the narrow array of active compounds.

The substituents $R^{1}$ on the phenyl ring were chosen in order to assess the influence that the electronic effects may have on the antimycobacterial activity. To ease the analysis, the compounds are divided in sub-series based on the identity and nature of $\mathrm{R}^{1}$. Thus, sub-series 1 comprises $\mathbf{1 - 5}$ with $\mathrm{R}^{1}(\mathrm{H})$; sub-series 2, 6-10 $\left(\mathrm{CH}_{3}\right)$, sub-series 3, 11-15 (Br); sub-series 4, 15-20 $\left(\mathrm{NO}_{2}\right)$ and sub-series 5, 21-25 $\left(\mathrm{CF}_{3}\right)$. Sub-series 1 is neutral, 2 is electron donating due to $\mathrm{CH}_{3}$ being an electron donating group (EDG) and 3-5 are electron withdrawing, accounting on the fact that $\mathrm{Br}, \mathrm{NO}_{2}$ and $\mathrm{CF}_{3}$ are electron withdrawing groups (EWGs) with $\mathrm{NO}_{2}, \mathrm{CF}_{3}$ and $\mathrm{Br}$ in order of decreasing EW. Sub-series 2 comprised four active compounds in GAST/Fe with $\mathrm{MIC}_{90}$ values varying in the $10-30 \mu \mathrm{M}$ range, while the others contain only 2 active compounds. For $4 \mathrm{C}$ chain compounds, the decreasing order of activity in GAST/Fe was $\mathbf{2 1}\left(\mathrm{CF}_{3}\right)$, $11(\mathrm{Br}), \mathbf{1}(\mathrm{H}), \mathbf{6}\left(\mathrm{CH}_{3}\right)$ and $16\left(\mathrm{NO}_{2}\right)$, and $7\left(\mathrm{CH}_{3}\right), \mathbf{2}(\mathrm{H})$, $17\left(\mathrm{NO}_{2}\right)$ and $22\left(\mathrm{CF}_{3}\right)$ for $6 \mathrm{C}$ side chain compounds. It could be noticed that within a sub-series the activity decreased as the chain length increased; however, no specific sub-series stood out. Thus, neither the electronic effect of substituents on the phenyl ring nor the length of the side chain of the triazole moiety dictated the activity of the compounds. Thus, structural specificity rather than the physical features (lipophilicity/chain length and electronic effect) governed the activity of these BZT derivatives. Individual compounds, such as $\mathbf{2 1}$ and $\mathbf{1 1}$ featuring $n$-butyl chain and bearing $\mathrm{CF}_{3}$ and $\mathrm{Br}$ EWGs, respectively, were identified as the most active compounds in the protein-free GAST/Fe medium. In protein-rich 7H9/ADC medium, however, $\mathbf{2 1}$ possessed weak activity, while $\mathbf{1 1}$ was completely inactive.

Furthermore, the charge distribution of organic molecules is influenced by the presence of halogen atoms. These can also affect binding interactions with biological systems (Zhang et al. 2017). Fluorine has an atomic radius comparable to hydrogen with higher electronegativity than other halogens and, hence, is known to have an effect on conformation, charge distribution, molecular interaction and pharmacokinetics (Gillis et al. 2015; O'Hagan 2008). The 
short chain (4C) halogen substituted compounds $\mathbf{1 1}$ and $\mathbf{2 1}$ were more active than their alkyl substituted counterparts $\mathbf{1}$ and 6.

Bioisosterism was also assessed. Indeed, a bioisostere is a molecule resulting from the exchange of an atom or of a group of atoms with an alternative, broadly similar atom or group of atoms. The objective of bioisosterism is the rational modification of a lead compound into safer and more clinically effective agents. The bioisosteric replacement can attenuate toxicity, modify activity of the lead and/ or alter pharmacokinetics of the lead.

The ability of a group of bioisosteres to elicit similar biological activity has been attributed to common physicochemical properties. Thus, specific physicochemical effects, such as electronegativity, steric size and lipophilicity, were quantitated to correlate these values to the observed biological activity. The substitution of hydrogen by fluorine is one of the more commonly employed monovalent isosteric replacements. Steric parameters for hydrogen and fluorine are similar with their van der Waal's radii being 1.2 and $1.35 \AA$, respectively (Leroux et al. 2008). Compound 6 is 4-Me (EDG) substituted, while 21 contained a larger $4-\mathrm{CF}_{3}$ (EWG). Both compounds have similar lipophilicity (cLogP 4.0), but 21 was 11-fold more antimycobacterially active than $\mathbf{6}$ in the albumin-free medium. Thus, the replacement of hydrogen with fluorine resulted in a safer hit $\left(\mathrm{IC}_{50}>100 \mu \mathrm{M}\right)$ by virtue of its greater electronegativity, while other parameters, such as steric size and lipophilicity, are maintained. This confirms fluorine as a bioisostere of hydrogen.

Overall, no synthesized compound was found to possess potency comparable or higher than any of the reference drugs of the study. However, both 21 and $\mathbf{1 1}$ are antimycobacterially promising hits with $\mathrm{MIC}_{90}<10 \mu \mathrm{M}$ (Katsuno et al. 2015). They were found to be nontoxic to the mammalian cells with $\mathrm{IC}_{50}>100 \mu \mathrm{M}$ as compared with $0.2 \mu \mathrm{M}$ of emetine.

\section{Conclusions}

A series of 25 BZT derivatives was synthesized in high yields in a single step by employing click chemistry. The compounds were evaluated in vitro for antimycobacterial activity against $M t b \mathrm{H} 37 \mathrm{Rv}$ strain using MABA assay in two media, BSA containing Middlebroth base 7H9 supplemented with ADC, and albumin-free GAST-Fe. Cytotoxicity was also assessed usingHEK-293 cells.

The antimycobacterial activity was growth medium dependent, with half of the compounds being active in the albumin-free medium, while most derivatives were inactive in the proteinaceous medium. The mechanism of action of the active compounds was not determined, and will require further work to elucidate.

For SAR purposes, only the $\mathrm{MIC}_{90}$ values in $\mathrm{GAST} / \mathrm{Fe}$ were considered. No realistic SAR could be drawn from this study due to the narrow array of active compounds. However, structural specificity of individual compounds rather than physicochemical properties in the series guided the activity. Compounds $\mathbf{2 1}$ and 11, featuring $n$-butyl side chain with trifluoromethyl and methyl substituent in para position on the phenyl ring, respectively, stood out as the most active with micromolar activities, although less potent than the standards, INH and RIF. Bioisomerism was also on display as the replacement of hydrogen in $p$-methyl of compound 6 with fluorine generated the $p$-trifluoromethyl containing hit 21 that was 11 times more active than $\mathbf{6}$.

These were also selective in their medium-dependent antibacterial actions as seen from the antimycobacterial assay. Both promising compounds had good selectivity towards mycobacteria, with SI values greater than 10, and thus stand as validated hits based on cellular potency and cytotoxicity criteria to be further investigated in the search for new antitubercular regimens. It will be interesting to assess the synergism of these antimycobacterial promising BZT derivatives in combination with higher PB affinity compounds using the same pathway of action.

Acknowledgements This work was funded by a South African National Research Foundation Grant to DDN'Da (UID 76443). The South African Medical Research Council is gratefully acknowledged for financial support of the antimycobacterial screening assays (SHIPMRC grant to DFW). The authors thank Dr D. Otto for NMR analysis and Dr JHL Jordaan for MS analysis. Isoniazid was generously donated by Aspen Pharmacare (Port Elizabeth, South Africa).

\section{Compliance with ethical standards}

Conflict of interest The authors declare that they have no conflict of interest.

Publisher's note Springer Nature remains neutral with regard to jurisdictional claims in published maps and institutional affiliations.

\section{References}

Abrahams GL, Kumar A, Savvi S, Hung AW, Wen S, Abell C, Barry III CE, Sherman DR, Boshoff HI, Mizrahi V (2012) Pathwayselective sensitization of Mycobacterium tuberculosis for targetbased whole-cell screening. Chem Biol 19(7):844-854

Ali AA, Gogoi D, Chaliha AK, Buragohain AK, Trivedi P, Saikia PJ, Gehlot PS, Kumar A, Chaturvedi V, Sarma D (2017) Synthesis and biological evaluation of novel 1,2,3-triazole derivatives as anti-tubercular agents. Bioorg Med Chem Lett 27:3698-3703

Amir A, Rana K, Arya A, Kapoor N, Kumar H, Siddiqui MA (2014) Mycobacterium tuberculosis H37Rv: in silico drug targets identification by metabolic pathways analysis. J Evol Biol 2014:8

Ballari MS, Herrera Cano N, Lopez AG, Wunderlin DA, Feresín GE, Santiago AN (2017) Green synthesis of potential antifungal 
agents: 2-benzyl substituted thiobenzoazoles. J Agric Food Chem 65(47):10325-10331

Belz T, Ihmaid S, Al-Rawi J, Petrovski S (2013) Synthesis characterization and antibacterial, antifungal activity of $\mathrm{N}$-(benzyl carbamoyl or carbamothioyl)-2-hydroxy substituted benzamide and 2-benzyl amino-substituted benzoxazines. Int $\mathbf{J}$ Med Chem 2013:436397

Boechat N, Ferreira VF, Ferreira SB, Ferreira MdLG, da Silva FdC, Bastos MM, Costa MdS, Lourenço MCS, Pinto AC, Krettli AU et al. (2011a) Novel 1,2,3-triazole derivatives for use against Mycobacterium tuberculosis H37Rv (ATCC 27294) strain. J Med Chem 54(17):5988-5999

Boechat N, Ferreira VF, Ferreira SB, Ferreira MdLG, da Silva FdC, Bastos MM, Costa MdS, Lourenço MCS, Pinto AC, Krettli AU et al. (2011b) Novel 1,2,3-triazole derivatives for use against Mycobacterium tuberculosis H37Rv (ATCC 27294) strain. J Med Chem 54:5988-5999

CDC (2018a) CDC I TB । Drug-resistant TB. Centers for Disease Control and Prevention, Atlanta, Georgia, US. https://www.cdc. gov/tb/topic/drtb/default.htm. Accessed 25 Jul 2019

CDC (2018b) Treatment for TB Disease. Centers for Disease Control and Prevention, Atlanta, Georgia, US. https://www.cdc.gov/tb/ topic/treatment/tbdisease.htm. Accessed 9 Oct 2018.

Cheng YJ, Liu ZY, Liang HJ, Fang CT, Zhang NN, Zhang TY, Yan M (2019) Discovery of (3-Benzyl-5-hydroxyphenyl)carbamates as new antitubercular agents with potent in vitro and in vivo efficacy. Molecules 24(10):pii: E2021

Collins L, Franzblau SG (1997) Microplate alamar blue assay versus BACTEC 460 system for high-throughput screening of compounds against Mycobacterium tuberculosis and Mycobacterium avium. Antimicrobial agents Chemother 41(5):1004-1009

Collins LA, Torrero MN, Franzblau SG (1998) Green fluorescent protein reporter microplate assay for high-throughput screening of compounds against Mycobacterium tuberculosis. Antimicrobial Agents Chemother 42(2):344-347

Courtens C, Risseeuw M, Caljon G, Cos P, Van Calenbergh S (2018) Acyloxybenzyl and alkoxyalkyl prodrugs of a fosmidomycin surrogate as antimalarial and antitubercular agents. ACS Med Chem Lett 9(10):986-989

Dai Z-C, Chen Y-F, Zhang M, Li S-K, Yang T-T, Shen L, Wang J-X, Qian S-S, Zhu H-L, Ye Y-H (2015) Synthesis and antifungal activity of 1,2, 3-triazole phenylhydrazone derivatives. Org Biomol Chem 13:477-486

De Voss JJ, Rutter K, Schroeder BG, Su H, Zhu Y, Barry CE, III. 2000. The salicylate-derived mycobactin siderophores of Mycobacterium tuberculosis are essential for growth in macrophages. In: Proceedings of the National Academy of Sciences of the United States of America. 97(3):1252-1257.

Dheer D, Singh V, Shankar R (2017) Medicinal attributes of 1,2,3triazoles: Current developments. Bioorg Chem 71:30-54

Dockal M, Carter DC, Ruker F (2000) Conformational transitions of the three recombinant domains of human serum albumin depending on pH. J Biol Chem 275(5):3042-3050

El Bissati K, Redel H, Ting L-M, Lykins JD, McPhillie MJ, Upadhya R, Woster PM, Yarlett N, Kim K, Weiss LM (2019) Novel synthetic polyamines have potent antimalarial activities in vitro and in vivo by decreasing intracellular spermidine and spermine concentrations. Front Cell Infect Microbiol 9(9):9

Fan Y-L, Wu J-B, Cheng X-W, Zhang F-Z, Feng L-S (2018) Fluoroquinolone derivatives and their anti-tubercular activities. Eur $\mathrm{J}$ Med Chem 146:554-563

Franzblau SG, DeGroote MA, Cho SH, Andries K, Nuermberger E, Orme IM, Mdluli K, Angulo-Barturen I, Dick T, Dartois V et al. (2012) Comprehensive analysis of methods used for the evaluation of compounds against Mycobacterium tuberculosis. Tuberculosis 92(6):453-488
Gallardo-Macias R, Kumar P, Jaskowski M, Richmann T, Shrestha R, Russo R, Singleton E, Zimmerman MD, Ho HP, Dartois V et al. (2019) Optimization of N-benzyl-5-nitrofuran-2-carboxamide as an antitubercular agent. Bioorg Med Chem Lett 29(4):601-606

Gillis EP, Eastman KJ, Hill MD, Donnelly DJ, Meanwell NA (2015) Applications of fluorine in medicinal chemistry. J Med Chem 58 (21):8315-8359

Gombar VK, Enslein K (1996) Assessment of n-octanol/water partition coefficient: when is the assessment reliable? J Chem Inf Comput Sci 36(6):1127-1134

Howson SE, Peter S (2010). Formation of benzyl azide from benzyl bromide; benzyl azide. ChemSpider - Synthetic. https://doi.org/ $10.1039 / \mathrm{SP} 408$

Jureen P, Werngren J, Toro JC, Hoffner S (2008) Pyrazinamide resistance and pncA gene mutations in Mycobacterium tuberculosis. Antimicrobial agents Chemother 52(5):1852-1854

Kandagal PB, Ashoka S, Seetharamappa J, Shaikh SM, Jadegoud Y, Ijare OB (2006) Study of the interaction of an anticancer drug with human and bovine serum albumin: spectroscopic approach. J Pharm Biomed Anal 41(2):393-399

Katsuno K, Burrows JN, Duncan K, Van Huijsduijnen RH, Kaneko T, Kita K, Mowbray CE, Schmatz D, Warner P, Slingsby BT (2015) Hit and lead criteria in drug discovery for infectious diseases of the developing world. Nat Rev Drug Disco 14:751

Kumar D, Beena, Khare G, Kidwai S, Tyagi AK, Singh R, Rawat DS (2014a) Synthesis of novel 1,2,3-triazole derivatives of isoniazid and their in vitro and in vivo antimycobacterial activity evaluation. Eur J Med Chem 81:301-313

Kumar K, Pradines B, Madamet M, Amalvict R, Benoit N, Kumar V (2014b) 1H-1,2,3-triazole tethered isatin-ferrocene conjugates: synthesis and in vitro antimalarial evaluation. Eur J Med Chem 87:801-804

Labadie GR, de la Iglesia A, Morbidoni HR (2011) Targeting tuberculosis through a small focused library of 1,2,3-triazoles. Mol Diversity 15(4):1017-1024

Leroux FR, Manteau B, Vors J-P, Pazenok S (2008) Trifluoromethyl ethers-synthesis and properties of an unusual substituent. Beilstein J Org Chem 4:13-13

Lipinski CA, Lombardo F, Dominy BW, Feeney PJ (1997) Experimental and computational approaches to estimate solubility and permeability in drug discovery and development settings. Adv Drug Deliv Rev 23:3-25

Machado D, Girardini M, Viveiros M, Pieroni M (2018) Challenging the drug-likeness dogma for new drug discovery in tuberculosis. Front Microbiol 9:1367

Mahata T, Kanungo A, Ganguly S, Modugula EK, Choudhury S, Pal SK, Basu G, Dutta S (2016) The benzyl moiety in a quinoxalinebased scaffold acts as a DNA intercalation switch. Angew Chem Int Ed Engl 55(27):7733-7736

Manetsch R, Krasiński A, Radić Z, Raushel J, Taylor P, Sharpless KB, Kolb HC (2004) In situ click chemistry: enzyme inhibitors made to their own specifications. J Am Chem Soc 126 (40):12809-12818

Massarotti A, Aprile S, Mercalli V, Del Grosso E, Grosa G, Sorba G, Tron GC (2014) Are 1,4- and 1,5-disubstituted 1,2,3-triazoles good pharmacophoric groups? ChemMedChem 9(11):2497-2508

Mohammed I, Kummetha IR, Singh G, Sharova N, Lichinchi G, Dang J, Stevenson M, Rana TM (2016) 1,2,3-triazoles as amide bioisosteres: discovery of a new class of potent HIV-1 Vif antagonists. J Med Chem 59:7677-7682

O'Hagan D (2008) Understanding organofluorine chemistry. An introduction to the C-F bond. Chem Soc Rev 37(2):308-319

Ollinger J, Bailey MA, Moraski GC, Casey A, Florio S, Alling T, Miller MJ, Parish T (2013) A dual read-out assay to evaluate the potency of compounds active against Mycobacterium tuberculosis. PLOS ONE 8(4):e60531 
Pop E, Oniciu DC, Pape ME, Cramer CT, Dasseux, J-LH. (2004) Lipophilicity parameters and biological activity in a series of compounds with potential cardiovascular applications. Croat Chem Acta 77:301-306

Ran D, Wu X, Zheng J, Yang J, Zhou H, Zhang M, Tang Y (2007) Study on the interaction between florasulam and bovine serum albumin. J Fluoresc 17(6):721-726

Rozwarski DA, Grant GA, Barton DH, Jacobs Jr. WR, Sacchettini JC (1998) Modification of the NADH of the isoniazid target (InhA) from Mycobacterium tuberculosis. Sciene 279(5347): 98-102

Sajja Y, Vanguru S, Vulupala HR, Nagarapu L, Perumal Y, Sriram D, Nanubolu JB (2017) Design, synthesis, and in vitro antituberculosis activity of benzo[6,7]cyclohepta[1,2-b]pyridine1,3,4-oxadiazole derivatives. Chem Biol Drug Des 90:496-500

Seetharamappa J, Kamat BP (2004) Spectroscopic studies on the mode of interaction of an anticancer drug with bovine serum albumin. Chem Pharm Bull 52(9):1053-1057

Seifert M, Catanzaro D, Catanzaro A, Rodwell TC (2015) Genetic mutations associated with isoniazid resistance in Mycobacterium tuberculosis: a systematic review. PLOS ONE 10(3): e0119628-e0119628

Shafi S, Mahboob Alam M, Mulakayala N, Mulakayala C, Vanaja G, Kalle AM, Pallu R, Alam MS (2012) Synthesis of novel 2mercapto benzothiazole and 1,2,3-triazole based bis-heterocycles: their anti-inflammatory and anti-nociceptive activities. Eur J Med Chem 49:324-333

Shin J-A, Lim Y-G, Lee K-H (2012) Copper-catalyzed azide-alkyne cycloaddition reaction in water using cyclodextrin as a phase transfer catalyst. J Org Chem 77(8):4117-4122

Singh A, Gut J, Rosenthal PJ, Kumar V (2017) 4-aminoquinolineferrocenyl-chalcone conjugates: synthesis and anti-plasmodial evaluation. Eur J Med Chem 125:269-277

Soares de Melo C, Feng TS, van der Westhuyzen R, Gessner RK, Street LJ, Morgans GL, Warner DF, Moosa A, Naran K, Lawrence $\mathrm{N}$ et al. (2015) Aminopyrazolo[1,5-a]pyrimidines as potential inhibitors of Mycobacterium tuberculosis: structure activity relationships and ADME characterization. Bioorg Med Chem 23(22):7240-7250

Speers AE, Adam GC, Cravatt BF (2003) Activity-based protein profiling in vivo using a copper(i)-catalyzed azide-alkyne [3 +2$]$ cycloaddition. J Am Chem Soc 125(16):4686-4687

Suresh N, Nagesh HN, Renuka J, Rajput V, Sharma R, Khan IA, Kondapalli Venkata, Gowri CS (2014) Synthesis and evaluation of 1-cyclopropyl-6-fluoro-1,4-dihydro-4-oxo-7-(4-(2-(4-substitute dpiperazin-1-yl)acetyl)piperazin-1-yl)quinoline-3-carboxylic acid derivatives as anti-tubercular and antibacterial agents. Eur J Med Chem 71:324-332

Swetha Y, Reddy ER, Kumar JR, Trivedi R, Giribabu L, Sridhar B, Rathod B, Prakasham RS (2019) Synthesis, characterization and antimicrobial evaluation of ferrocene-oxime ether benzyl 1H-1,2,3-triazole hybrids. New J Chem 43(21):8341-8351

Tahghighi A, Karimi S, Rafie Parhizgar A, Zakeri S (2018) Synthesis and antiplasmodial activity of novel phenanthroline derivatives: an in vivo study. Iran J Basic Med Sci 21(2):202-211

Tian J, Liu J, Zhang J, Hu Z, Chen X (2003) Fluorescence studies on the interactions of barbaloin with bovine serum albumin. Chem Pharm Bull 51(5):579-582

Tornøe CW, Christensen C, Meldal M (2002) Peptidotriazoles on solid phase: [1,2,3]-triazoles by regiospecific copper(I)-catalyzed 1,3dipolar cycloadditions of terminal alkynes to azides. J Org Chem 67(9):3057-3064

WHO (2016) WHO treatment guidelines for drug-resistant tuberculosis 2016 update. World Health Organization, Geneva, Switzerland.

WHO (2018a) Infectious diseases. World Health Organization, Geneva, Switzerland. https://www.who.int/topics/infectious_diseases/ en/. Accessed 2 Jul 2019

WHO (2018b) Global TB Report 2018. World Health Organization, Geneva, Switzerland. https://www.who.int/tb/publications/globa 1_report/en/. Accessed 4 Jul 2019

Wilson CR, Gessner RK, Moosa A, Seldon R, Warner DF, Mizrahi V, Soares de Melo C, Simelane SB, Nchinda A, Abay E et al. (2017) Novel antitubercular 6-dialkylaminopyrimidine carboxamides from phenotypic whole-cell high throughput screening of a softfocus library: structure-activity relationship and target identification studies. J Med Chem 60(24):10118-10134

Yee D, Valiquette C, Pelletier M, Parisien I, Rocher I, Menzies D (2003) Incidence of serious side effects from first-line antituberculosis drugs among patients treated for active tuberculosis. Am J Respir Crit Care Med 167(11):1472-1477

Zaw MT, Emran NA, Lin Z (2018) Mutations inside rifampicinresistance determining region of $\mathrm{rpoB}$ gene associated with rifampicin-resistance in Mycobacterium tuberculosis. J Infect Public Health 11(5):605-610

Zhang N-n, Liu Z-y, Liang J, Tang Y-x, Qian L, Gao Y-m, Zhang T-y, Yan M (2018) Design, synthesis, and biological evaluation of $\mathrm{m}-$ amidophenol derivatives as a new class of antitubercular agents. MedChemComm 9(8):1293-1304

Zhang S, Xu Z, Gao C, Ren Q-C, Chang L, Lv Z-S, Feng L-S (2017) Triazole derivatives and their anti-tubercular activity. Eur J Med Chem 138:501-513 